\title{
Signatures of the correlation hole in total and partial cross sections
}

\author{
T. Gorin* and T. H. Seligman \\ Centro de Ciencias Físicas, University of Mexico (UNAM), C. P. 62210 Cuernavaca, México
}

\begin{abstract}
In a complex scattering system with few open channels, say a quantum dot with leads, the correlation properties of the poles of the scattering matrix are most directly related to the internal dynamics of the system. We may ask how to extract these properties from an analysis of cross sections. In general this is very difficult, if we leave the domain of isolated resonances. We propose to consider the cross correlation function of two different elastic or total cross sections. For these we can show numerically and to some extent also analytically a significant dependence on the correlations between the scattering poles. The difference between uncorrelated and strongly correlated poles is clearly visible, even for strongly overlapping resonances.

PACS number(s): 05.45.Mt, 03.65.Nk
\end{abstract}

\section{Introduction}

Starting from Bohr's compound nucleus suggestion, the idea of considering the dynamics in the interaction region almost separately from the scattering process has been proven very successful in different fields. Wigner's R-matrix method [1] gives the formal background to the separation of internal dynamics and "free" motion in the channel space. Based on this idea we study whether chaoticity or integrability of the internal dynamics can be detected in the scattering data. For this purpose we apply Fourier transform techniques, which have proved successful in spectral analysis [2, 3, 1], to total and partial cross sections.

To build the scattering ensembles, we shall assume that there are no correlations between channel space and the internal structure. This assumption is usually well fulfilled for systems with topological chaos, while it is often not fulfilled for integrable systems [0]. Nevertheless, we use this assumption in order to compare the chaotic and regular case in a direct and minimally biased way. Any differences we then find are basis independent and minimal in the sense that correlations would usually increase the dissimilarity to the chaotic case. Therefore, we use orthogonally invariant random matrix models to describe the internal structure. For the chaotic case the choice is obviously the Gaussian orthogonal ensemble (GOE) if time reversal symmetry is conserved. Following Berry and Tabor $[6]$ we associate integrability with a random Poissonian spectrum, thus excluding harmonic oscillators explicitly. For this case the Poisson orthogonal ensemble (POE) was proposed some years ago [7]. The case of time reversal symmetry breaking can be treated analogously using the unitary ensembles, but this will not be discussed in the present paper.

We consider three different absorption regimes. For weak coupling the resonances are isolated, and conventional spectral analysis is satisfactory; if we wish we may add an analysis of the widths. Then follows what is usually called strong absorption, where we find overlapping resonances, but where the individual transmissions from all channels are considerably smaller than one. Next we have the case where the transmissions are close to one. To differentiate the latter two regimes, we shall speak of strong transmission in this case. It corresponds to the semiclassical limit, where tunneling effects become negligible.

For level spectra as well as for intensity spectra, Fourier transform methods and the so called "correlation hole" have been very successful tools to identify the effects of integrability and chaos [2, 3, 4, 8]. Yet it is not clear to what extent such an analysis remains useful, when the resonances are no longer isolated. An exact theory is only available for two-point functions of S-matrix elements in the GOE case. This so called Verbaarschot-Weidenmüller-Zirnbauer (VWZ) integral [9] allows to calculate correlation functions of total cross sections by means of the optical theorem. In contrast to that, we have no such theory in the POE case or for correlation functions of partial cross sections. In these cases, one had to fall back on the so called "Breit-Wigner" approximation [10], which becomes valid in the weak coupling limit. To extend the validity of this approximation

\footnotetext{
*Present address: Theoretical Quantumdynamics, Albert-Ludwigs-Universität, Hermann-Herder Str. 3, 79104 Freiburg. E-mail: Thomas.Gorin@physik.uni-freiburg.de
} 
we take advantage of the well known fact that the Satchler transmission matrix 11] or, in absence of direct reactions as in our case, the transmission coefficients are the only way in which the coupling strength influences the physically relevant quantities. By using the transmission coefficients rather than the coupling constants as input, which amounts to a rescaling, we are able to extend the validity of this approximation to the regime of strong absorption, where the resonances are overlapping, but the absorption in each channel is still moderate.

We perform numerical simulations with two purposes: to check the range of validity of the rescaled BreitWigner formalism, and to find situations, which show significant differences between GOE and POE. We will concentrate on Fourier transforms of auto and cross correlation functions, and we shall see that in particular for the latter the differences are in some cases very promising. In particular we find that cross correlations between cross sections from different channels show strong signatures.

In Sec. 2 we present the model we use. In Sec. 3 we introduce the correlation functions of S-matrix elements and cross sections, our basic tool for the statistical analysis of the scattering systems. Next we discuss the "rescaled Breit-Wigner" approximation in Sec. 1. It allows to get results in closed form for the correlation

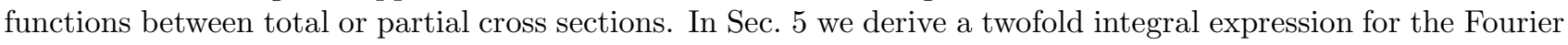
transform of the VWZ integral [9]. This simplifies the numerical treatment considerably, and it is used in Sec. 6. to test the validity of the rescaled Breit-Wigner approximation. After these theoretical considerations, we turn to the numerical study of the two scattering ensembles, the POE and the GOE. This is done in Sec. 6, which is divided into two subsections. The first deals with correlation functions between total cross sections, and the second with correlation functions between partial cross sections. Section 8 contains a short summary.

\section{The scattering ensembles}

We wish to construct scattering ensembles for the two contrary cases, where the dynamics in the interaction region is predominantly integrable, or completely chaotic. We do this under the assumption that the scattering system may be separated into an internal part restricted to a finite interaction region and an external part described by some superintegrable Hamiltonian. Though some complexity may show up in the coupling of the two parts also, i.e. in the mismatch of channel functions and internal functions, the dominant part of the complexity should be contained in the internal part. Then the subsystem that describes the complex internal dynamics has a discrete spectrum, such that its statistical properties can be modeled with an appropriate random matrix ensemble. In order to construct the scattering ensembles, we fix the external part and use standard techniques, originally introduced to describe nuclear compound reactions [12, 13, 14, to assign to each element from the random matrix ensemble a scattering matrix. Thus we obtain a set of scattering matrices provided with the measure inherited from the original random matrix ensemble. For simplicity we will denote the scattering ensembles obtained from the GOE and the POE by the same names whenever there is no danger for confusion.

To be more precise, we consider complex scattering systems with many, possibly overlapping, resonances, where the S-matrix can be cast into the following form:

$$
S(E)=1-\mathrm{i} V^{\dagger} \frac{1}{E-H} V, \quad H=H_{\text {int }}-\frac{\mathrm{i}}{2} V V^{\dagger} .
$$

Here $H_{\text {int }}$ is a real, symmetric $N \times N$ matrix which describes the internal dynamics, and $V$ is a real $N \times M$ matrix, describing the coupling to the $M$ channels. The matrix $H$ is the so called effective Hamiltonian 15$]$. In order to arrive at Eq. (1) it is assumed that the coupling matrix elements between channel states and internal eigenstates are energy independent [14]. Furthermore one should either neglect the direct reactions or perform an Engelbrecht-Weidenmüller transformation [16] if it is necessary to take them into account. The effective Hamiltonian $H$ can be diagonalized, such that its eigenvalues $\tilde{E}_{j}=E_{j}-\mathrm{i} \Gamma_{j} / 2$ give the positions and widths of the resonances if they are isolated. In the eigenbasis of $H$, the S-matrix elements can be written as

$$
\begin{aligned}
S_{a b}(E) & =\delta_{a b}-\mathrm{i} \sum_{j=1}^{N} \frac{\tilde{V}_{j a} \tilde{V}_{j b}}{E-\tilde{E}_{j}}, \\
\tilde{V} & =A^{T} V, \quad A^{T} H A=\operatorname{diag}\left(\tilde{E}_{j}\right) .
\end{aligned}
$$

This equation shows that the complex poles of the S-matrix are precisely the $N$ eigenvalues of $H$. If the coupling matrix elements are small enough, their real parts are well approximated by the discrete levels of $H_{\text {int }}$, whereas their imaginary parts are given by the diagonal elements of $V V^{\dagger}$. This amounts to the Breit-Wigner approximation, which results from applying first order perturbation theory to the effective Hamiltonian $H$. 
In this paper we consider two scattering ensembles: the GOE and the POE. Both are invariant with respect to orthogonal transformations. Hence in the eigenbasis of $H_{\text {int }}$ the $M$ channel vectors are random orthogonal vectors. In practice, we use independent random vectors with Gaussian distributed components for $V$, which are orthogonal only up to order $\mathcal{O}\left(N^{-1}\right)$. However, as we used relatively large matrices $N=300$, the violation of the orthogonal invariance had certainly no noticeable effect on our numerical results. Hence, for both ensembles the nonzero eigenvalues of $V V^{\dagger}$ (given by the norm squared of the column vectors), and the level density of $H_{\text {int }}$ are the only independent parameters.

In the GOE case, the elements of the diagonal matrix $H_{\text {int }}$ are distributed according to the joint probability distribution of the GOE spectrum [17], so that for large $N$, the level density approaches the semicircle distribution. In the POE case, the elements are independently distributed, and in principle the level density can be of any form. Our main objective is the distinction between both ensembles, based on the observation of correlations. Hence we find it convenient to use the semicircle distribution in the POE case also.

The main theoretical tool for the calculation of correlation functions will be the rescaled Breit-Wigner approximation, introduced in Sec. 1 . In fact, it can be applied in much more general situations. This is of particular importance for partially integrable scattering systems, where the assumption of orthogonal invariance does often not hold. Then the distribution of the matrix elements of $V$ are typically very different from simple uncorrelated Gaussian distributions.

In cases as they are studied here, the openness of the scattering system is commonly described, borrowing the terminology from the so called "optical model" ( $c f$. [14] and references therein), which was originally developed to describe nuclear compound reactions with two well separated time scales. Consider the partial cross section in appropriate units, which is given by: $\sigma_{a b}=\left|\delta_{a b}-S_{a b}\right|^{2}$. Then one defines the optical partial cross section as $\sigma_{a b}^{\text {opt }}=\left|\delta_{a b}-\left\langle S_{a b}\right\rangle\right|^{2}$, where the different time scales are used to obtain a well defined average S-matrix $\left\langle S_{a b}\right\rangle$ by averaging over an appropriately chosen energy window. Here $\left\langle S_{a b}\right\rangle$ is simply defined by the ensemble average, avoiding in this way any arbitrariness. The openness of the scattering system is then characterized by so called "transmission coefficients," defined for each entrance channel $a$ :

$$
T_{a}=\left\langle\sigma_{\mathrm{tot}}^{(a)}\right\rangle-\sigma_{\mathrm{opt}}^{(a)}=1-\sum_{c=1}^{M}\left|\left\langle S_{a c}\right\rangle\right|^{2},
$$

where $\sigma_{\text {tot }}^{(a)}=\sum_{c=1}^{M} \sigma_{a c}$ is the total cross section, and $\sigma_{\mathrm{opt}}^{(a)}=\sum_{c=1}^{M} \sigma_{a c}^{\mathrm{opt}}$ is the total optical cross section, with respect to the entrance channel $a$. The unitarity of the S-matrix leads to:

$$
\sigma_{\text {tot }}^{(a)}=2\left(1-\operatorname{Re} S_{a a}\right),
$$

which is sometimes called the "optical theorem."

The scattering ensembles defined above, i.e. the GOE and the POE, are completely characterized by the average level distance $d=(N \rho)^{-1}$ in the center of the spectrum of $H_{\text {int }}$, and the variance of the coupling matrix elements $\left\langle V_{i a}^{2}\right\rangle$, which are independent of $i$ due to orthogonal invariance. From those we define the following dimensionless coupling parameters:

$$
\kappa_{a}=\frac{\pi}{2 d}\left\langle V_{i a}^{2}\right\rangle .
$$

Here and in what follows, the brackets $\langle\ldots\rangle$ stand for the ensemble average. As discussed in the Appendix, the average S-matrix is diagonal. Its diagonal elements, the transmission coefficients, and the coupling parameters are related to each other:

$$
\left\langle S_{a a}\right\rangle=\frac{1-\kappa_{a}}{1+\kappa_{a}} \Leftrightarrow T_{a}=\frac{4 \kappa_{a}}{\left(1+\kappa_{a}\right)^{2}},
$$

which is, however, true in the center of the spectrum only (a more detailed discussion is given in the Appendix).

As mentioned in the introduction, we distinguish three different regimes. Now, these can be defined more quantitatively in terms of the transmission coefficients: The first is the weak absorption regime, where the resonances are still well separated, so that $\sum_{a=1}^{M} T_{a} \ll 1$. Next comes the regime of strong absorption, where the resonances overlap, but the transmission from each channel is still small: $\sum_{a=1}^{M} T_{a}>1, \forall a: T_{a} \ll 1$. Note, that this implies in particular, that the number of channels $M$ is large. Finally we have the regime of strong transmission: $\forall a: T_{a} \lesssim 1$ where the transmissions in all channels are close to one. 


\section{Correlation functions of total and partial cross sections}

Correlation functions are our principal tool for the statistical analysis of total and partial cross sections. We distinguish between autocorrelation functions, where one cross section is correlated with itself, and cross correlation functions, where two different cross sections are correlated with each other. First we define the correlation functions in general, in order to introduce our notation. Then we use the optical theorem, to relate the correlation function of two total cross sections to one of corresponding S-matrix elements. For the latter, the VWZ integral [9] provides the exact result in the GOE case. In the POE case an exact result exists only in the one channel case [18], and we use the rescaled Breit-Wigner approximation there. For partial cross sections, no exact theory exists at all. In this case, we first use the so called "diagonal approximation" to express the partial cross sections in such a form, that the rescaled Breit-Wigner approximation can be applied.

Dealing with matrix ensembles, it is convenient to define the correlation functions as ensemble averages, rather then energy averages. Therefore, we eventually have to face the ergodicity question [19], which is unclear in the POE case. Note, however, that in quantum dot experiments ensemble averages may actually be the relevant ones [20]. We calculate the correlation functions always in the center of the spectrum (see Appendix), where we set $E=0$. Given then two complex functions $f$ and $g$ of the energy, we define the correlation function as follows:

$$
C[f, g](\omega)=\left\langle f\left(\frac{-\omega d}{2}\right) g\left(\frac{\omega d}{2}\right)\right\rangle-\left\langle f\left(\frac{-\omega d}{2}\right)\right\rangle\left\langle g\left(\frac{\omega d}{2}\right)\right\rangle .
$$

Here $d$ is the mean level distance in the center of the spectrum of $H_{\mathrm{int}}$ which is assumed to be constant on the scale where we expect correlations. Note that, there is no unfolding involved. The mean level distance $d$ simply serves as a convenient energy scale. For the discrete spectrum of some random Hamiltonian: $f(E)=\sum_{i} \delta\left(E-E_{i}\right)$, the autocorrelation function becomes: $C\left[f, f^{*}\right](\omega)=1+\delta(\omega)-Y_{2}(\omega)$, where $Y_{2}$ is the two-point cluster function as defined in Ref. 17].

We will mainly analyze the correlations in the time domain, and by consequence deal with the Fourier transforms of correlation functions. In general, we denote the Fourier transform of a given function of the energy $f(E)$ by:

$$
\hat{f}(t)=\mathcal{F}[f](t)=\int \mathrm{d} \omega \mathrm{e}^{2 \pi \mathrm{i} \omega t} f(\omega d)
$$

where the Fourier transform is taken with respect to the energy measured in units of $d$, and the factor $2 \pi$ in the exponent assures proper normalization. For two spectral functions $f$ and $g$, the following relation holds:

$$
\hat{C}[f, g](t)=\frac{1}{L}\left\{\left\langle\hat{f}^{\prime}(-t) \hat{g}^{\prime}(t)\right\rangle-\left\langle\hat{f}^{\prime}(-t)\right\rangle\left\langle\hat{g}^{\prime}(t)\right\rangle\right\},
$$

where the functions $f^{\prime}(x)$ and $g^{\prime}(x)$ are equal to $f(x)$ and $g(x)$ inside the interval $|x / d|<L / 2$ and zero outside, and the limits $N, L \rightarrow \infty, L / N \rightarrow 0$ are taken. Equation (9) is based on the convolution theorem [21] applied to the fluctuating parts of the spectral functions $f$ and $g$, where the convolution integral is expressed as a correlation function as in Eq. (7) assuming stationarity. The limit $N, L \rightarrow \infty$ is necessary, to allow the correlation function to go to zero quickly enough, so that the Fourier integral of the correlation function is well defined. The limit $L / N \rightarrow 0$ serves to obtain stationarity in the interval where the correlation function is calculated. In particular, the average level (or resonance) density and the average S-matrix should not vary noticeably in this interval. Equation (9) is used in the numerical calculation of the correlation functions. It turned out, that $L=N / 2, N=300$ already gives well converged results.

Note that we measure the energy in units of the mean level spacing $d$. As a result, the argument of a correlation function is dimensionless, and so is the argument of its Fourier transform. Nevertheless the latter is denoted by $t$, as its significance is still time - though measured in units of $d^{-1}$.

Total cross sections Consider the correlation function of two total cross sections $\sigma_{\text {tot }}^{(a)}$ and $\sigma_{\text {tot }}^{(b)}$ with possibly different entrance channels $a$ and $b$. As the total cross sections depend linearly on the respective diagonal S-matrix elements [see Eq. (田)] the correlation function can be expressed as follows:

$$
\begin{aligned}
C\left[\sigma_{\mathrm{tot}}^{a}, \sigma_{\mathrm{tot}}^{b}\right] & =C\left[S_{a a}+S_{a a}^{*}, S_{b b}+S_{b b}^{*}\right] \\
& =2 \operatorname{Re}\left(C\left[S_{a a}, S_{b b}\right]+C\left[S_{a a}, S_{b b}^{*}\right]\right) \\
& =2 \operatorname{Re} C\left[S_{a a}, S_{b b}^{*}\right] .
\end{aligned}
$$


The correlation function of nonconjugated elements $C\left[S_{a a}, S_{b b}\right]$ vanishes [22]. Relation (10) is essential, as it relates experimentally accessible quantities to analytical results [9, 23, 18]. For the Fourier transform of Eq. (10) we get:

$$
\begin{aligned}
\hat{C}\left[\sigma_{\text {tot }}^{a}, \sigma_{\text {tot }}^{b}\right](t) & =2 \mathcal{F} \operatorname{Re} C\left[S_{a a}, S_{b b}^{*}\right](t) \\
& =\hat{C}\left[S_{a a}, S_{b b}^{*}\right](t)+\hat{C}\left[S_{a a}, S_{b b}^{*}\right](-t) \\
& =\hat{C}\left[S_{a a}, S_{b b}^{*}\right](t),
\end{aligned}
$$

where it is assumed that $t>0$. Then $\hat{C}\left[S_{a a}, S_{b b}^{*}\right](-t)$ vanishes, because of its negative argument [24] (see also Sec. 4.1). For the sake of brevity let us think of a correlation function and its Fourier transform as a single object represented in the energy domain or in the time domain, respectively, and call it simply "correlation function" or "C-function" in either case.

Partial cross sections Partial cross sections are given by $\sigma_{a b}=\left|\delta_{a b}-S_{a b}\right|^{2}$. The theoretical treatment of correlation functions of partial cross sections is complicated by the fact that one has to average over a product of four S-matrix elements. The insertion of the S-matrix elements as given in Eq. (2) leads to a double sum of resonance terms. As an exact analytical treatment seems to be impossible, we employ the diagonal approximation, which consists in retaining the diagonal terms of the double sum only. This is justified for sufficiently weak coupling and leads to:

$$
\sigma_{a b} \approx \sigma_{a b}^{\prime}=\sum_{j=1}^{N} \frac{\gamma_{j a} \gamma_{j b}}{\left(E-E_{j}\right)^{2}+\Gamma_{j}^{2} / 4}, \quad \gamma_{j a}=\left|\tilde{V}_{j a}\right|^{2} .
$$

The r.h.s. can be written as the imaginary part of a function $\int_{a b}(E)$, which has the same pole structure as the S-matrix:

$$
\begin{aligned}
\sigma_{a b}^{\prime}(E) & =-2 \operatorname{Im} \int_{a b}(E), \\
\int_{a b}(E) & =\sum_{j=1}^{N} \frac{1}{\Gamma_{j}} \frac{\gamma_{j a} \gamma_{j b}}{E-E_{j}+\mathrm{i} \Gamma_{j} / 2} .
\end{aligned}
$$

Due to the linear relation between the diagonal approximation $\sigma_{a b}^{\prime}$ and the spectral function $\int_{a b}$, we may again express correlation functions of the former by corresponding correlation functions of the latter. In fact, we have:

$$
C\left[\sigma_{a b}, \sigma_{c d}\right](\omega) \approx C\left[\sigma_{a b}^{\prime}, \sigma_{c d}^{\prime}\right](\omega)=2 \operatorname{Re} C\left[\int_{a b}, \int_{c d}^{*}\right](\omega) .
$$

It remains to calculate the correlation function of $\int_{a b}$ and $\int_{c d}^{*}$. This will be done in the following section using the rescaled Breit-Wigner approximation. In order to calculate the Fourier transform of Eq. (14), we note that the pole structure of $\int_{a b}(E)$ is the same as that of the true S-matrix, so that $\hat{C}\left[\int_{a b}, \int_{c d}^{*}\right](-t)$ is again zero (for $t>0)$. Therefore, we obtain:

$$
\hat{C}\left[\sigma_{a b}, \sigma_{c d}\right](t) \approx \hat{C}\left[\sigma_{a b}^{\prime}, \sigma_{c d}^{\prime}\right](t)=\hat{C}\left[\int_{a b}, \int_{c d}^{*}\right](t) .
$$

\section{Rescaled Breit-Wigner approximation}

In this section we calculate the correlation function of two arbitrary S-matrix elements using the Breit-Wigner approximation, followed by a phenomenological rescaling procedure. To this end Eq. (9) is used, which means that we first calculate the Fourier transform of the respective S-matrix elements (this can be done exactly), and then we average over the resonance parameters $\left\{E_{j}, \Gamma_{j}, \tilde{V}_{j 1}, \ldots, \tilde{V}_{j M}\right\}_{1 \leq j \leq N}$. The average over the real parts of the S-matrix poles $\left\{E_{j}\right\}$ can still be done in a formally exact manner, but then we have to use the approximation mentioned above, in order to finish the task. To obtain the correlation functions of partial cross sections, the same steps have to be done with the matrix elements $\int_{a b}$ instead [see Eq. (13)]. 


\subsection{Formally exact treatment}

Here, we do all those steps of the calculation which are exact. We first calculate the Fourier transform of one S-matrix element, using its pole expansion (2). For $t>0$, we get:

$$
\begin{aligned}
\hat{S}_{a b}(-t) & =\frac{-\mathrm{i}}{d} \sum_{j=1}^{N} \tilde{V}_{j a} \tilde{V}_{j b} \int_{-L / 2}^{L / 2} \mathrm{~d} \omega \frac{\mathrm{e}^{-2 \pi \mathrm{i} \omega t}}{\omega-\frac{E_{j}-\mathrm{i} \Gamma_{j} / 2}{d}} \\
& =\frac{-\mathrm{i}}{d} \sum_{j=1}^{L} \tilde{V}_{j a} \tilde{V}_{j b} \mathrm{e}^{-2 \pi \mathrm{i} E_{j} t / d} \mathrm{e}^{-\pi \Gamma_{j} t / d} .
\end{aligned}
$$

Here it was used, that the poles with real parts outside the integration region do not contribute to the Fourier transform, and for those inside, it is well justified to extend the integration up to infinity because $\Gamma_{j} / L \ll 1$.

In the same way, we may obtain an analogous expression for $\hat{S}_{c d}(t)$. The Fourier transforms of the average S-matrix elements are taken into account in the numerical calculation only. Here by contrast, we notice that the average S-matrix elements are almost constant in the integration interval, which means that in the limit $N, L \rightarrow \infty$ their Fourier transforms will become $\delta$-functions situated at $t=0$. Thus they play no role in the current calculation which is restricted to $t>0$. Note that we may define $\hat{C}\left[S_{a b}, S_{c d}^{*}\right](0)=\lim _{t \rightarrow 0} \hat{C}\left[S_{a b}, S_{c d}^{*}\right](t)$. Inserting the expressions for $\hat{S}_{a b}(-t)$ and $\hat{S}_{c d}^{*}(t)$ into Eq. (9) and ignoring the Fourier transforms of the average S-matrix elements, we obtain:

$$
\begin{aligned}
\hat{C}\left[S_{a b}, S_{c d}^{*}\right](t) & =\frac{1}{d^{2} L} \sum_{j, k=1}^{L} \tilde{V}_{j a} \tilde{V}_{j b} \tilde{V}_{k c}^{*} \tilde{V}_{k d}^{*} \mathrm{e}^{-2 \pi \mathrm{i}\left(E_{j}-E_{k}\right) t / d} \mathrm{e}^{-\pi\left(\Gamma_{j}+\Gamma_{k}\right) t / d} \\
& =\frac{1}{d^{2}}\left\{\left\langle\tilde{V}_{j a} \tilde{V}_{j b} \tilde{V}_{j c}^{*} \tilde{V}_{j d}^{*} \mathrm{e}^{-2 \pi \Gamma_{j} t / d}\right\rangle+(L-1)\left\langle\tilde{V}_{j a} \tilde{V}_{j b} \tilde{V}_{k c}^{*} \tilde{V}_{k d}^{*} \mathrm{e}^{-\pi\left(\Gamma_{j}+\Gamma_{k}\right) t / d} \mathrm{e}^{-2 \pi \mathrm{i}\left(E_{j}-E_{k}\right) t / d}\right\rangle\right\} .
\end{aligned}
$$

As the ensemble average is invariant for any permutation of the resonance indices, the double sum can be evaluated. In the final expression the resonance indices $j \neq k$ are arbitrary. Note that $\hat{C}\left[S_{a b}, S_{c d}^{*}\right](t)$ vanishes for $t<0$, because in this case both Fourier transforms $\hat{S}_{a b}(-t)$ and $\hat{S}_{c d}^{*}(t)$ vanish, as can be easily seen by applying the residue theorem ( $c f$. also 24]).

At last, we average formally over the real parts $\left\{E_{j}\right\}$ of the S-matrix poles. For fixed values of the partial amplitudes and the total widths, the average over $-L \exp \left[-2 \pi \mathrm{i}\left(E_{j}-E_{k}\right) t / d\right]$ for $L \rightarrow \infty$ gives the two-point form factor [17] of the random sequence $\left\{E_{j}\right\}$, which we denote by $\tilde{b}_{2}(t)$. In general, $\tilde{b}_{2}(t)$ still depends on the parameters fixed. In the weak coupling limit, however, the positions of the resonances on the one hand, and the partial amplitudes and total widths on the other hand, become statistically independent, so that $\tilde{b}_{2}(t)$ converges to the two-point form factor of the closed system. After all we may write:

$$
\hat{C}\left[S_{a b}, S_{c d}^{*}\right](t)=\frac{1}{d^{2}}\left\{\left\langle\tilde{V}_{j a} \tilde{V}_{j b} \tilde{V}_{j c}^{*} \tilde{V}_{j d}^{*} \mathrm{e}^{-2 \pi \Gamma_{j} t / d}\right\rangle-\left\langle\tilde{V}_{j a} \tilde{V}_{j b} \tilde{V}_{k c}^{*} \tilde{V}_{k d}^{*} \mathrm{e}^{-\pi\left(\Gamma_{j}+\Gamma_{k}\right) t / d} \tilde{b}_{2}(t)\right\rangle\right\}
$$

This is so far an exact but rather formal result. However, it clearly shows that the correlation function is no direct measure for spectral correlations. The first term in Eq. (18), which may very well dominate the correlation function, contains the parameters of only one single resonance. Therefore, it cannot describe correlations between different resonances that are the ones we are really interested in. It is typically a monotonously decreasing function, where the decay is governed by the average width of the resonances. It is the second term in Eq. (18), which contains the parameters of two different resonances. It vanishes completely if there are no correlations between them. For our investigations it is important to find situations, where the first term is relatively small, so that one may retrieve as much information as possible on the correlations between different resonances.

\subsection{Approximation}

In order to evaluate the remaining averages in Eq. (18) we have to introduce some approximations. To this end, consider the weak coupling limit: $\forall a: \kappa_{a} \rightarrow 0$. Using first order perturbation theory in the expression for the S-matrix, Eqs. (11) and (2), we get the pure Breit-Wigner approximation for the S-matrix:

$$
S_{a b}(E) \approx \delta_{a b}-\mathrm{i} \sum_{j=1}^{N} \frac{V_{j a} V_{j b}}{E-\varepsilon_{j}+\mathrm{i} \sum_{c} V_{j c}^{2} / 2} .
$$


This amounts to make the following replacements in the pole expansion of the S-matrix (2):

$$
\tilde{V}_{j a} \rightarrow V_{j a}, \quad \Gamma_{j} \rightarrow \sum_{c=1}^{M} V_{j a}^{2}, \quad E_{j} \rightarrow \varepsilon_{j},
$$

where $\varepsilon_{j}$ are the eigenvalues of $H_{\mathrm{int}}$. Hence, in order to obtain the correlation functions in the Breit-Wigner approximation, we simply do the same replacements in Eq. (18). Then the partial amplitudes become real uncorrelated Gaussian random variables, the total widths become simple functions of the partial amplitudes, and $\tilde{b}_{2}(t)$ becomes the two-point form factor $b_{2}(t)$ of the spectrum of $H_{\mathrm{int}}$ :

$$
\hat{C}\left[S_{a b}, S_{c d}^{*}\right](t) \approx \frac{1}{d^{2}}\left\{\left\langle V_{j a} V_{j b} V_{j c} V_{j d} \mathrm{e}^{-2 \pi \sum_{c} V_{j c}^{2} t / d}\right\rangle-\left\langle V_{j a} V_{j b} V_{k c} V_{k d} \mathrm{e}^{-\pi \sum_{c}\left(V_{j c}^{2}+V_{k c}^{2}\right) t / d}\right\rangle b_{2}(t)\right\} .
$$

The remaining Gaussian averages are relatively simple, so that in many cases the respective correlation function can be calculated in closed form. Note that the averages are different from zero, only if all partial amplitudes appear in even powers.

Unfortunately, the pure Breit-Wigner approximation drifts quickly away from the exact result, as the coupling to the continuum increases. The following phenomenological procedure improves the approximation considerably. It consists in rescaling the variance of the partial amplitudes as follows:

$$
\left\langle V_{j a}^{2}\right\rangle \rightarrow \frac{\left\langle V_{j a}^{2}\right\rangle}{\left(1+\kappa_{a}\right)^{2}} .
$$

We call the result the "rescaled Breit-Wigner" approximation. As shown in Ref. [25], it leads to partial fluctuating cross sections of the Hauser-Feshbach type [26], showing elastic enhancements of 2 (GOE case) and 3 (POE case) in agreement with earlier theoretical results [28, 27, 29]. The occurrence of Ericson fluctuations [30] with the correlation length $\Gamma_{\mathrm{C}}=d \sum_{a=1}^{M} T_{a} /(2 \pi)$ is also correctly described. In Sec. $\mathrm{f}$ it is shown that in the time domain this approximation gives results for the correlation functions between S-matrix elements which become exact as $t \rightarrow 0$.

Note that the rescaled Breit-Wigner approximation can be applied in a wide range of situations, while it conserves the simplicity of the pure Breit-Wigner approximation. This makes it a valuable tool for the statistical description of complex scattering systems. This approximation can be justified to some extent with the following reasoning: It is well known, that the properties of a scattering system are determined by the Satchler transmission matrix [11], if the entire process is occurring on two different time scales, one associated with direct processes and one with long time processes also called compound processes in Nuclear physics. In the absence of direct reactions or after an Engelbrecht-Weidenmüller transformation [16], this implies the dependence on the transmission coefficients only. They are directly related to the variances of the partial amplitudes as mentioned above. The rescaled approximation thus implies that we use the transmission coefficients of the system rather than the coupling constants. This can be viewed as a nonperturbative input, or as using phenomenological parameters.

\section{Total cross sections}

According to Eq. (11), the C-function between two total cross sections is equal to the C-function between the respective diagonal S-matrix elements. Hence $\hat{C}\left[\sigma_{\text {tot }}^{(a)}, \sigma_{\text {tot }}^{(b)}\right](t)$ is given by the r.h.s. of Eq. (18) setting $b=a$ and $d=c=b$. In order to calculate this C-function in the rescaled Breit-Wigner approximation, we use the Eqs. (5) and (6) to express the ensemble averages as integrals over normalized squared amplitudes $g_{1}, \ldots, g_{M}$ :

$$
\hat{C}\left[\sigma_{\text {tot }}^{(a)}, \sigma_{\text {tot }}^{(b)}\right](t) \approx T_{a} T_{b}\left\{\left\langle g_{a} g_{b} \mathrm{e}^{-G t}\right\rangle-\left\langle g_{a} \mathrm{e}^{-G t / 2}\right\rangle\left\langle g_{b} \mathrm{e}^{-G t / 2}\right\rangle b_{2}(t)\right\},
$$

with $G=\sum_{c=1}^{M} T_{c} g_{c}$. The normalized squared amplitudes $g_{c}$ are distributed as the random variables $V_{i a}^{2} /\left\langle V_{i a}^{2}\right\rangle$, i.e. they are Porter-Thomas distributed [31]:

$$
p\left(g_{c}\right)=\frac{1}{\sqrt{2 \pi g_{c}}} \mathrm{e}^{-g_{c} / 2}, \quad c=1, \ldots, M .
$$

Now, the remaining averages in Eq. (23) can be calculated easily [25]. For the auto C-function of the total cross section we get:

$$
\hat{C}\left[\sigma_{\text {tot }}^{(a)}\right](t) \approx T_{a}^{2}\left\{3\left(1+2 T_{a} t\right)^{-5 / 2} \prod_{c \neq a}\left(1+2 T_{c} t\right)^{-1 / 2}-\left(1+T_{a} t\right)^{-3} \prod_{c \neq a}\left(1+T_{c} t\right)^{-1} b_{2}(t)\right\} .
$$


Note, that this result is very similar to the result of Ref. 32] for the spectral autocorrelation function of the photodissociation cross section in weakly coupled chaotic systems (there the rescaling is used also, though without mentioning it; note that these authors obtain a rescaled formula for their specific case directly as an approximation to exact results [33]). For the cross C-function of two different total cross sections, we get:

$$
\begin{aligned}
\hat{C}\left[\sigma_{\text {tot }}^{(a)}, \sigma_{\text {tot }}^{(b)}\right](t) \approx T_{a} T_{b}\left\{\left(1+2 T_{a} t\right)^{-3 / 2}\left(1+2 T_{b} t\right)^{-3 / 2} \prod_{c \neq a, b}\left(1+2 T_{c} t\right)^{-1 / 2}\right. \\
\left.\quad-\left(1+T_{a} t\right)^{-2}\left(1+T_{b} t\right)^{-2} \prod_{c \neq a, b}\left(1+T_{c} t\right)^{-1} b_{2}(t)\right\} .
\end{aligned}
$$

In what follows, we assume that all coupling strengths are equal: $\forall a: \kappa=\kappa_{a}, T=T_{a}$. For convenience, the numerical analysis in Sec. 7 is restricted to this case only. The Eqs. (25) and (26) simplify considerably and can be combined into a single one:

$$
\hat{C}\left[\sigma_{\text {tot }}^{(a)}, \sigma_{\text {tot }}^{(b)}\right] \approx T^{2}\left\{\left(1+2 \delta_{a b}\right)(1+2 T t)^{-2-M / 2}-(1+T t)^{-2-M} b_{2}(t)\right\} .
$$

To lowest order in the transmission coefficient $T$ the r.h.s. becomes $T^{2}\left[1+2 \delta_{a b}-b_{2}(t)\right]$ in agreement with results on intensity weighted stick spectra [4, 8] and the asymptotic behavior of the exact analytical result for the GOE case (see Eq. (59) in Sec. 5). The difference in the C-function at small times between GOE and POE is known as the correlation hole. In order to quantify it, we use its size at $t=0$ relative to the maximal size of the C-function in the POE case. From Eq. (27) it follows that the correlation hole is $1 / 3$ in the case of the auto C-function $(a=b)$, while it is one in the case of the cross C-function $(a \neq b)$.

\section{Partial cross sections}

According to Eq. (15), the C-function of two partial cross sections in the diagonal approximation is equal to the C-function of the matrix elements $\int_{a b}$ and $\int_{c d}^{*}$, defined in Eq. (13). Following the same lines as in the case of the true S-matrix, we arrive at the following expression for the latter C-function:

$$
\hat{C}\left[\int_{a b}, \int_{c d}^{*}\right](t) \approx \frac{4 \pi^{2}}{d^{2}}\left\{\left\langle\frac{\gamma_{1 a} \gamma_{1 b} \gamma_{1 c} \gamma_{1 d}}{\Gamma_{1}^{2}} \mathrm{e}^{-2 \pi \Gamma_{1} t / d}\right\rangle-\left\langle\frac{\gamma_{1 a} \gamma_{1 b}}{\Gamma_{1}} \frac{\gamma_{2 c} \gamma_{2 d}}{\Gamma_{2}} \mathrm{e}^{-\pi\left(\Gamma_{1}+\Gamma_{2}\right) t / d} \tilde{b}_{2}(t)\right\rangle\right\},
$$

which is the analog of Eq. (18). To this expression we can apply the rescaled Breit-Wigner approximation. The partial widths $\gamma_{j a}$, are replaced by Porter-Thomas distributed random variables with average value $\left\langle\gamma_{j a}\right\rangle=$ $T_{a} d /(2 \pi)$, the total widths become the sums of the partial widths, and the two-point form factor $\tilde{b}_{2}(t)$ becomes the two-point form factor of the spectrum of $H_{\text {int }}$. Thus we get:

$$
C\left[\sigma_{a b}, \sigma_{c d}\right](t) \approx T_{a} T_{b} T_{c} T_{d}\left\{\left\langle\frac{g_{a} g_{b} g_{c} g_{d}}{G^{2}} \mathrm{e}^{-G t}\right\rangle-\left\langle\frac{g_{a} g_{b}}{G} \mathrm{e}^{-G t / 2}\right\rangle\left\langle\frac{g_{c} g_{d}}{G} \mathrm{e}^{-G t / 2}\right\rangle b_{2}(t)\right\},
$$

where $G=\sum_{c} T_{c} g_{c}$. The ensemble average can be performed, using the following identities:

$$
\begin{aligned}
G^{-1} \mathrm{e}^{-\alpha G} & =\int_{\alpha}^{\infty} \mathrm{d} \alpha^{\prime} \mathrm{e}^{-\alpha^{\prime} G}, \\
G^{-2} \mathrm{e}^{-\alpha G} & =\int_{\alpha}^{\infty} \mathrm{d} \alpha^{\prime} \int_{\alpha^{\prime}}^{\infty} \mathrm{d} \alpha^{\prime \prime} \mathrm{e}^{-\alpha^{\prime \prime} G}, \quad \alpha>0 .
\end{aligned}
$$

Exchanging the integration on $\alpha^{\prime}$ and $\alpha^{\prime \prime}$ with the ensemble average, we can do the ensemble average analytically, and we are only left with the integrals over the auxiliary variables $\alpha^{\prime}$ and $\alpha^{\prime \prime}$. With the exception of some special cases, those integrals have to be evaluated numerically (for details, see Ref. [25]). In the case of equal coupling strengths, however, we obtain the following analytical expression:

$$
\hat{C}\left[\sigma_{a b}, \sigma_{c d}\right](t) \approx \frac{T^{2}}{(2+M)^{2}}\left\{\frac{(1+2 / M)^{2}}{(1+6 / M)(1+4 / M)} A(1+2 T t)^{-2-M / 2}-B(1+T t)^{-2-M} b_{2}(t)\right\} .
$$

Here, $A$ and $B$ depend on the actual combinations of partial widths in Eq. (29), i.e. whether any of the channels involved coincide or not. The following cases occur in the present paper: 
- The autocorrelation function:

$\hat{C}\left[\sigma_{a b}\right]: A=9+96 \delta_{a b}, B=1+8 \delta_{a b}$.

- The cross correlation function between two different elastic cross sections:

$\hat{C}\left[\sigma_{a a}, \sigma_{b b}\right]: A=9, B=9$.

- The cross correlation function between an elastic and an inelastic cross section:

$\hat{C}\left[\sigma_{a a}, \sigma_{a b}\right]: A=15, B=3$.

According to Eq. (31), the coefficients $A$ and $B$ determine the relative depth of the correlation hole in the limit of many channels $M \rightarrow \infty$. The prefactor $(1+2 / M)^{2} /[(1+6 / M)(1+4 / M)]$ in front of $A$ may, however, lead to a considerably deeper correlation hole, as long as $M$ is not too large.

For $M \rightarrow \infty$ and $M T$ fixed, the algebraic decay in Eq. (31) turns into an exponential one:

$$
(1+2 T t)^{-2-M / 2} \sim(1+T t)^{-2-M} \sim \exp (-M T t) .
$$

This means, that we obtain Ericson fluctuations 30 in this limit. The reason for the occurrence of Ericson fluctuations can be understood from Eq. (29): Only in the case of many channels and small transmission coefficients, does the central limit theorem lead to negligible fluctuations of the total width around its average value. Then we may treat $G$ as a constant, which leads immediately to the expected exponential decay. Note that this implies that Ericson fluctuations are no reliable signature for chaotic scattering, because the central limit theorem may work, even if the partial amplitudes are not Gaussian distributed, thus leading again to Ericson fluctuations. In such a situation, the deviation from the exponential decay due to $b_{2}(t)$, would be the only reliable signature of the chaotic dynamics.

\section{The Fourier transform of the VWZ integral}

In this section we derive a general formula for the Fourier transform of the VWZ integral [9]. This allows to obtain exact results for the correlation functions of total cross sections in the GOE case. We need these results, in order to check the accuracy of the rescaled Breit-Wigner approximation. Apart from that it will turn out, that numerically it is much easier to calculate the Fourier transform than the original VWZ integral:

$$
\begin{aligned}
C\left[S_{a b}, S_{c d}^{*}\right](\omega)= & \frac{1}{8} \iint_{0}^{\infty} \mathrm{d} \lambda_{1} \mathrm{~d} \lambda_{2} \int_{0}^{1} \mathrm{~d} \lambda \frac{\lambda(1-\lambda)\left|\lambda_{1}-\lambda_{2}\right| \mathrm{e}^{-\mathrm{i} \pi \omega\left(\lambda_{1}+\lambda_{2}+2 \lambda\right)}}{\sqrt{\lambda_{1}\left(1+\lambda_{1}\right) \lambda_{2}\left(1+\lambda_{2}\right)}\left(\lambda+\lambda_{1}\right)^{2}\left(\lambda+\lambda_{2}\right)^{2}} \\
& \times \prod_{e=1}^{M} \frac{1-T_{e} \lambda}{\sqrt{\left(1+T_{e} \lambda_{1}\right)\left(1+T_{e} \lambda_{2}\right)}}\left\{\delta_{a b} \delta_{c d} \Delta_{a} \Delta_{c}+\left(\delta_{a c} \delta_{b d}+\delta_{a d} \delta_{b c}\right) \Pi_{a b}\right\}, \\
\Delta_{a}= & T_{a} \sqrt{1-T_{a}}\left(\frac{\lambda_{1}}{1+T_{a} \lambda_{1}}+\frac{\lambda_{2}}{1+T_{a} \lambda_{2}}+\frac{2 \lambda}{1-T_{a} \lambda}\right), \\
\Pi_{a b}= & T_{a} T_{b}\left(\frac{\lambda_{1}\left(1+\lambda_{1}\right)}{\left(1+T_{a} \lambda_{1}\right)\left(1+T_{b} \lambda_{1}\right)}+\frac{\lambda_{2}\left(1+\lambda_{2}\right)}{\left(1+T_{a} \lambda_{2}\right)\left(1+T_{b} \lambda_{2}\right)}+\frac{2 \lambda(1-\lambda)}{\left(1-T_{a} \lambda\right)\left(1-T_{b} \lambda\right)}\right) .
\end{aligned}
$$

The main interest in the VWZ integral has been the calculation of average fluctuating cross sections [22, 34, 35, 36], which corresponds to the case $\omega=0$ in Eq. (33). Only a few papers treat the $\omega$-dependence of the VWZ integral [24, 37, 38], and even then the analysis was usually restricted to particular limits such as $\omega \rightarrow 0(t \rightarrow \infty$ in the time domain), or many channels: $M \rightarrow \infty$ and Ericson fluctuations. Quite often the existence of the correlation hole was simply ignored.

In the present analysis, the correlation hole and its dependence on the coupling strengths is of great importance. It is needed to distinct regular from chaotic dynamics. Therefore, we will analyze the Fourier transform of the VWZ integral in some detail. We will also observe the behavior of the correlation hole in the limits: $t \rightarrow 0$ (Sec. 5.1) and $\forall a: T_{a} \rightarrow 0$ (Sec. 5.2).

Let us start our derivation by applying the Fourier transform (8) to Eq. (33). We exchange the Fourier integration on $\omega$ with the integrals on $\lambda, \lambda_{1}$, and $\lambda_{2}$. For the Fourier integration we then simply need to calculate:

$$
\begin{array}{r}
\int \mathrm{d} \omega \exp \left\{2 \pi \mathrm{i} \omega\left[t-\left(\lambda_{1}+\lambda_{2}+2 \lambda\right) / 2\right]\right\} \\
=\delta\left[t-\left(\lambda_{1}+\lambda_{2}+2 \lambda\right) / 2\right] .
\end{array}
$$


The $\delta$-function can be used to remove the $\lambda$-integral. To the remaining double integral, the following transformations are applied:

$$
\begin{array}{r}
\lambda_{1}, \lambda_{2} \rightarrow r=\frac{\lambda_{1}+\lambda_{2}}{2}, \quad s=\lambda_{2}-\lambda_{1} \\
\text { followed by } s \rightarrow x=r^{2}-s^{2} / 4 .
\end{array}
$$

This leads to:

$$
\hat{C}\left[S_{a b}, S_{c d}^{*}\right](t)=\frac{1}{4} \int_{\max (0, t-1)}^{t} \mathrm{~d} r(t-r)(r+1-t) \prod_{e=1}^{M}\left[1-T_{e}(t-r)\right] U(r),
$$

where

$$
\begin{aligned}
U(r) & =2 \int_{0}^{r^{2}} \mathrm{~d} x \frac{\delta_{a b} \delta_{c d} \Delta_{a} \Delta_{c}+\left(\delta_{a c} \delta_{b d}+\delta_{a d} \delta_{b c}\right) \Pi_{a b}}{\left(t^{2}-r^{2}+x\right)^{2} \sqrt{x(x+2 r+1)} \sqrt{\prod_{e=1}^{M}\left(1+2 T_{e} r+T_{e}^{2} x\right)}}, \\
\Delta_{a} & =2 T_{a} \sqrt{1-T_{a}}\left(\frac{r+T_{a} x}{1+T_{a}\left(2 r+T_{a} x\right)}+\frac{t-r}{1-T_{a}(t-r)}\right), \text { and } \\
\Pi_{a b} & =2 T_{a} T_{b}\left(\frac{T_{a} T_{b} x^{2}+\left[T_{a} T_{b} r+\left(T_{a}+T_{b}\right)(r+1)-1\right] x+r(2 r+1)}{\left(1+2 T_{a} r+T_{a}^{2} x\right)\left(1+2 T_{b} r+T_{b}^{2} x\right)}+\frac{(t-r)(r+1-t)}{\left[1-T_{a}(t-r)\right]\left[1-T_{b}(t-r)\right]}\right) .
\end{aligned}
$$

In order to remove the $1 / \sqrt{x}$-singularity in the integrand of $U(r)$, we substitute consecutively:

$$
x=(y-1) b / 2, \quad y=\left(z+z^{-1}\right) / 2, \quad z=2 u+1 \quad \text { where } \quad b=1+2 r .
$$

This gives:

$$
U(r)=4 \int_{0}^{r} \frac{\mathrm{d} u}{2 u+1} \frac{\delta_{a b} \delta_{c d} \Delta_{a} \Delta_{c}+\left(\delta_{a c} \delta_{b d}+\delta_{a d} \delta_{b c}\right) \Pi_{a b}}{\left(t^{2}-r^{2}+x\right)^{2} \sqrt{\prod_{e=1}^{M}\left(1+2 T_{e} r+T_{e}^{2} x\right)}}, \quad x=\frac{b u^{2}}{2 u+1} .
$$

The Eqs. (36) and (41) together with the Eqs. (38) and (39) form our final result for the correlation function $\hat{C}\left[S_{a b}, S_{c d}^{*}\right](t)$ (it is understood, to replace $x$ by $b u^{2} /(2 u+1)$ wherever it occurs). One may readily use these formulas for numerical calculations. All problematic singularities have been removed from the integration region. Our result if followed by a fast Fourier transformation back to the energy domain, may even be a quite efficient way of computing the original VWZ integral.

The Figs. 1 and 2 show examples of the Fourier-transformed auto and cross correlation functions of the total cross section, calculated with the help of Eq. (36), etc. The coupling strength for all channels was fixed to the value $\kappa=0.1$, while the number of channels $M$ was varied. The qualitative features of the C-functions are almost the same for any $M$. In the first place, $M$ determines the falloff at large times. Note, however, that in the case of extremely small total transmission, we would obtain a "true" correlation hole at $t=0$ (i.e. a positive slope). See also the related discussion below Eq. (27)] which is concerned with the rescaled Breit-Wigner result. In all, it permits us to restrict the numerical studies in Sec. 7 to the case $M=2$.

Figure 1 shows the autocorrelation function divided by $T^{2}$, which starts at $t=0$ with the value 2 , independent of the number of channels. It decays with time, the larger the number of channels, the faster. This is very reasonable, as the decay must be related to the total transmission $\sum_{a} T_{a}=M T$. Note that there is no qualitative signature (positive slope at $t=0$ ) of the correlation hole.

In Fig. 2 the cross correlation function is shown, which starts at $t=0$ with zero. It increases linearly with time, and it seems that now the slope is independent of the number of channels. At large times, the cross C-function must go to zero in the same way as the auto C-function shown in Fig. 1. Hence, after reaching a maximum that decreases and moves to the left with increasing $M$, the cross C-function decays to zero. Therefore, we see a clear signature of the correlation hole.

To conclude this section we will consider two different asymptotic limits: First we will prove, that for $t \rightarrow 0$ the asymptotic behavior of the various C-functions is independent of $M$. Second we will show with very high numerical precision, that for small transmission coefficients, $\forall a: T_{a} \rightarrow 0$, the cross C-functions coincide (if properly normalized) with the two-level form factor $b_{2}(t)$ for the GOE. 


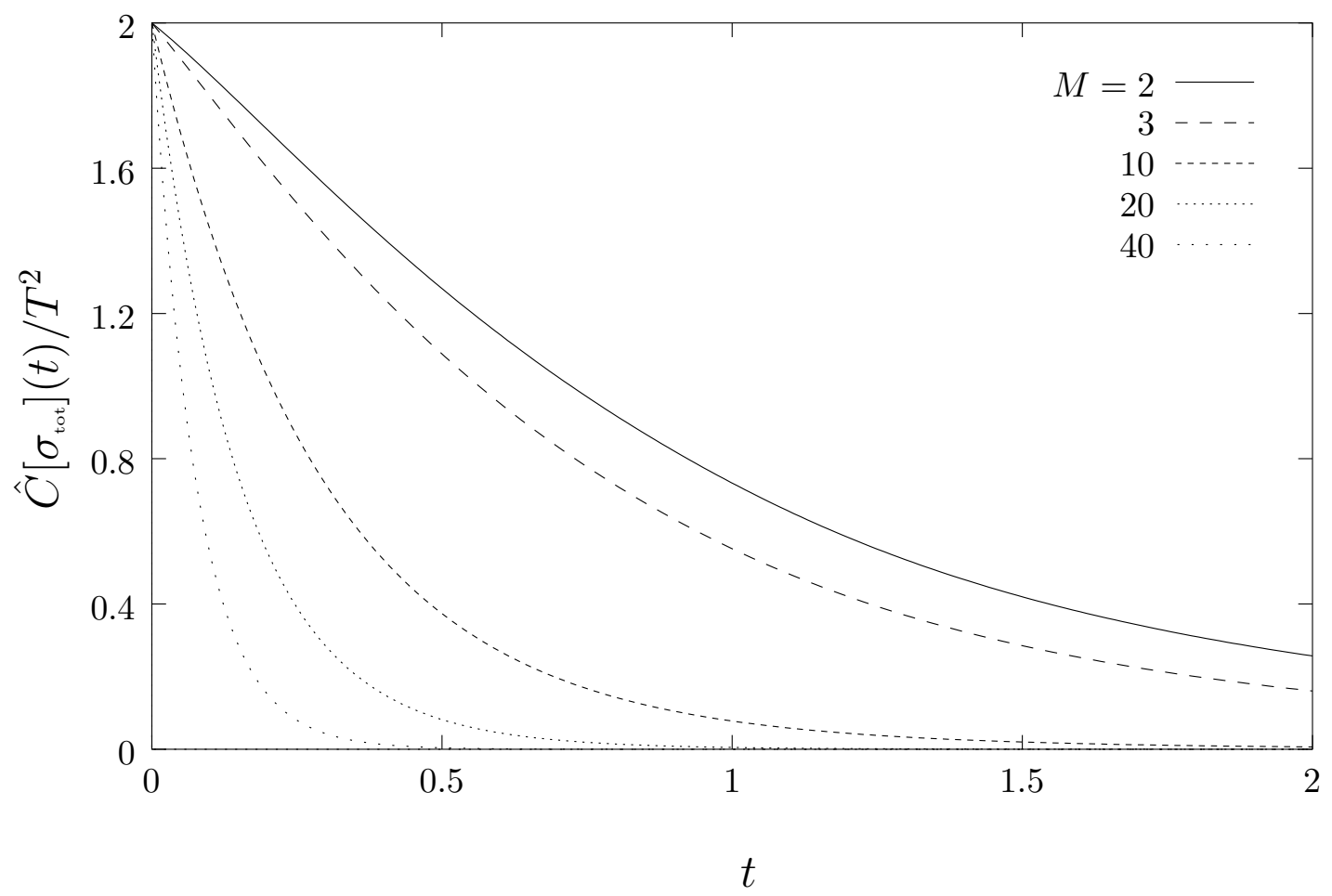

Figure 1: The exact result (36) for the autocorrelation function $\hat{C}\left[\sigma_{\text {tot }}\right](t)$ divided by $T^{2}$. While $\kappa=0.1$ is kept fixed, the number of channels is varied: $M=2$ (solid line), $M=3$ (long dashed line), $M=10$ (short dashed line), $M=20$ (dotted line), and $M=40$ (long dotted line).

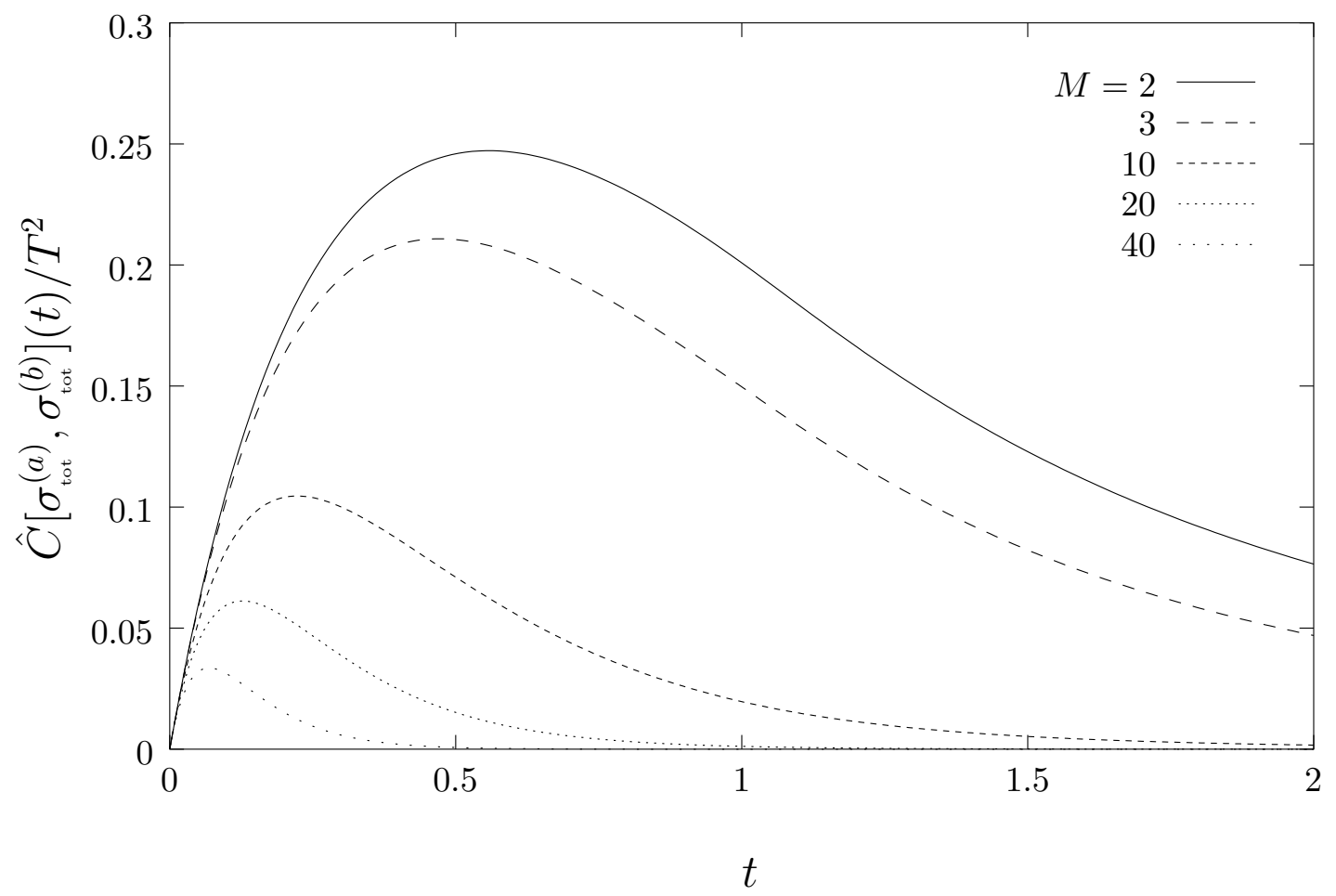

Figure 2: As in Fig. 1, but for the cross correlation function $\hat{C}\left[\sigma_{\text {tot }}^{a}, \sigma_{\text {tot }}^{b}\right](t)$ divided by $T^{2}$. 


\subsection{The limit of small times}

In the limit $t \rightarrow 0$, the Eq. (36) can be approximated by:

$$
\begin{aligned}
\hat{C}\left[S_{a b}, S_{c d}^{*}\right](t) & \sim \frac{t^{2}}{4} \int_{0}^{1} \mathrm{~d} \varrho(1-\varrho) U(t \varrho) \\
& =\frac{1}{4} \int_{0}^{1} \mathrm{~d} \varrho(1-\varrho) \tilde{U}(\varrho),
\end{aligned}
$$

where $\tilde{U}(\varrho)=t^{2} U(t \rho)$. Hence setting $r=t \rho$ in Eq. (41) the following limits can by taken: $b \sim 1,2 u+1 \sim 1$, and $x \sim u^{2}$. Then substituting $u=t v$ we get:

$$
\begin{gathered}
\tilde{U}(\varrho) \sim \frac{4}{t} \int_{0}^{1} \mathrm{~d} v\left[\delta_{a b} \delta_{c d} \Delta_{a} \Delta_{c}+\left(\delta_{a c} \delta_{b d}+\delta_{a d} \delta_{b c}\right) \Pi_{a b}\right] \\
\div\left[\left(1-\varrho^{2}+v^{2}\right)^{2} \sqrt{\prod_{e=1}^{M}\left(1+2 T_{e} t \varrho+T_{e}^{2} t^{2} v^{2}\right)}\right] \\
\sim \frac{4}{t} \int_{0}^{1} \mathrm{~d} v \frac{\delta_{a b} \delta_{c d} \Delta_{a} \Delta_{c}+\left(\delta_{a c} \delta_{b d}+\delta_{a d} \delta_{b c}\right) \Pi_{a b}}{\left(1-\varrho^{2}+v^{2}\right)^{2}} .
\end{gathered}
$$

To lowest order the Eqs. (38) and (39) simplify also:

$$
\begin{aligned}
\Delta_{a} & \sim 2 T_{a} \sqrt{1-T_{a}} t\left(1+T_{a} t v^{2}\right) \sim 2 T_{a} \sqrt{1-T_{a}} t \\
\Pi_{a b} & \sim 2 T_{a} T_{b} t\left[1+\left(T_{a}+T_{b}-1\right) t v^{2}\right] \sim 2 T_{a} T_{b} t .
\end{aligned}
$$

This shows, that if the $\mathrm{C}$-function contains the $\Delta$-term only, we get a linear increase starting at $t=0$, whereas if it contains the $\Pi$-term also, we get a finite value at $t=0$, but no valid value for the slope. The slope in the first case and the finite value in the second are both determined by the same integral $I_{1}$ :

$$
\begin{aligned}
\hat{C}\left[S_{a b}, S_{c d}^{*}\right](t) & \sim\left[4 \delta_{a b} \delta_{c d} T_{a} T_{c} \sqrt{\left(1-T_{a}\right)\left(1-T_{c}\right)} t+2\left(\delta_{a c} \delta_{b d}+\delta_{a d} \delta_{b c}\right) T_{a} T_{b}\right] I_{1}, \\
I_{1} & =\int_{0}^{1} \mathrm{~d} \varrho(1-\varrho) \int_{0}^{1} \frac{\mathrm{d} v}{\left(1-\varrho^{2}+v^{2}\right)^{2}}=\int_{0}^{1} \mathrm{~d} \varrho(1-\varrho)\left[\frac{1}{2 p^{3}} \arctan \left(\frac{\varrho}{p}\right)+\frac{\varrho}{2 p^{2}}\right]
\end{aligned}
$$

where $p^{2}=1-\varrho^{2}$. Splitting $I_{1}$ into two parts and substituting $x=\arctan (\varrho / p)$ in the first one, we can evaluate $I_{1}$ analytically:

$$
I_{1}=\frac{1}{2} \int_{0}^{\pi / 2} \mathrm{~d} x \frac{x}{1+\sin x}+\frac{1}{2} \int_{0}^{1} \mathrm{~d} \varrho \frac{\varrho}{1+\varrho}=\frac{\ln 2}{2}+\frac{1-\ln 2}{2}=\frac{1}{2}
$$

In order to contain the $\Delta$-term only, the C-function must be of the form: $\hat{C}\left[S_{a a}, S_{b b}^{*}\right]$ with $a \neq b$. Then its asymptotic behavior is:

$$
\hat{C}\left[S_{a a}, S_{b b}^{*}\right] \sim 2 T_{a} T_{b} \sqrt{\left(1-T_{a}\right)\left(1-T_{b}\right)} t .
$$

If the C-function is of the form $\hat{C}\left[S_{a b}, S_{a b}^{*}\right]=\hat{C}\left[S_{a b}, S_{b a}^{*}\right]$, then it contains the $\Pi$-term also. In this case we get:

$$
\hat{C}\left[S_{a b}, S_{a b}^{*}\right](0)=\left(1+\delta_{a b}\right) T_{a} T_{b} .
$$

These results confirm our observations in Fig. 1 1 and Fig. 2. The cross correlation function of two total cross sections vanishes at $t=0$. This holds for all values of the transmission coefficient. Moreover, the slope only depends on the transmission coefficients of the entrance and exit channels, and thus does not change if the number of channels is changed. In contrast to that, for the auto correlation function of a total cross section we get: $\hat{C}\left[\sigma_{\text {tot }}^{(a)}\right](0)=2 T_{a}^{2}$. Again this value does not depend on the number of channels.

Comparing the asymptotic results (49) and (50) to the rescaled Breit-Wigner approximation (27) we find agreement in lowest order - for the slope in the case of cross correlation functions, and for the value at $t=0$ in the case of autocorrelation functions. 


\subsection{The limit of small transmission coefficients}

In the case $\forall a: T_{a} \rightarrow 0$, the Eqs. (36) and (41) simplify to:

$$
\hat{C}\left[S_{a b}, S_{c d}^{*}\right](t) \sim \frac{1}{4} \int_{\max (0, t-1)}^{t} \mathrm{~d} r(t-r)(r+1-t) U(r),
$$

where

$$
U(r) \sim 4 \int_{0}^{r} \frac{\mathrm{d} u}{2 u+1} \frac{\delta_{a b} \delta_{c d} \Delta_{a} \Delta_{c}+\left(\delta_{a c} \delta_{b d}+\delta_{a d} \delta_{b c}\right) \Pi_{a b}}{\left(t^{2}-r^{2}+\frac{b u^{2}}{2 u+1}\right)^{2}} .
$$

In the same limit, the expression for $\Delta_{a}(38)$ and the one for $\Pi_{a b}(39)$ become:

$$
\begin{aligned}
\Delta_{a} & \sim 2 T_{a} t \\
\Pi_{a b} & \sim 2 T_{a} T_{b}\left[(t-r)(r+1-t)+r(2 r+1)-\frac{b u^{2}}{2 u+1}\right] .
\end{aligned}
$$

Defining the two integrals:

$$
\begin{aligned}
& I_{2}=\int_{0}^{r} \mathrm{~d} u \frac{2 u+1}{\left[\left(t^{2}-r^{2}\right)(2 u+1)+b u^{2}\right]^{2}} \\
& I_{3}=\int_{0}^{r} \mathrm{~d} u \frac{u^{2}}{\left[\left(t^{2}-r^{2}\right)(2 u+1)+b u^{2}\right]^{2}},
\end{aligned}
$$

the asymptotic limit of $U(r)$ can be written as

$$
U(r) \sim 8\left\{2 \delta_{a b} \delta_{c d} T_{a} T_{c} t^{2} I_{2}+\left(\delta_{a c} \delta_{b d}+\delta_{a d} \delta_{b c}\right) T_{a} T_{b}\left[\left(r^{2}+2 t r+t-t^{2}\right) I_{2}-b I_{3}\right]\right\} .
$$

In order to keep the discussion short, we shall consider cross C-functions of the type $\hat{C}\left[S_{a a}, S_{b b}^{*}\right], a \neq b$ only. Then we only need to calculate $I_{2}$. Defining $p^{2}=t^{2}-r^{2}$ and $R^{2}=b-p^{2}, I_{2}$ can be written as follows:

$$
\begin{aligned}
I_{2} & =-\frac{1}{2 p} \partial_{p} \int_{0}^{r} \frac{\mathrm{d} u}{b u^{2}+2 p^{2} u+p^{2}} \\
& =-\frac{1}{2 p} \partial_{p} \frac{1}{p R}\left(\arctan \frac{b r+p^{2}}{p R}-\arctan \frac{p}{R}\right) \\
& =\frac{R^{2}-p^{2}}{2 p^{3} R^{3}}\left(\arctan \frac{b r+p^{2}}{p R}-\arctan \frac{p}{R}\right)-\frac{r(r+1)}{2 t^{2} p^{2} R^{2}} \\
& =\frac{R^{2}-p^{2}}{2 p^{3} R^{3}} \arctan \frac{r R}{(r+1) p}+\frac{r(r+1)}{2 t^{2} p^{2} R^{2}} .
\end{aligned}
$$

Inserting Eq. (57) into Eq. (51), we get the following result for $\hat{C}\left[S_{a a}, S_{b b}^{*}\right]$ :

$$
\frac{1}{T_{a} T_{b}} \hat{C}\left[S_{a a}, S_{b b}^{*}\right](t) \sim 4 t^{2} \int_{\max (0, t-1)}^{t} \mathrm{~d} r(t-r)(r+1-t) I_{2},
$$

where $I_{2}$ is given in Eq. (58). We have evaluated Eq. (59) numerically and compared to the expected result $1-b_{2}(t)$, for the discrete GOE-spectrum. The difference is of the order of the machine precision $\left(\approx 10^{-11}\right)$ for any value of $t$.

\section{Test of the rescaled Breit-Wigner approximation}

The aim of the present paper is actually twofold. In the first place, we analyze correlation functions of total and partial cross sections, to search for signatures of the correlation hole, in particular in the regime of overlapping resonances. In the second place we wish to establish the rescaled Breit-Wigner approximation as a simple and general tool for the analysis of correlations in different scattering situations. This section is devoted to the latter, whereas the the correlation hole will be studied in Sec. 7.

We use the rescaled Breit-Wigner approximation in those cases, where no exact theory is available, i.e. for C-functions of partial cross sections (where we need the additional diagonal approximation) and for C-functions 


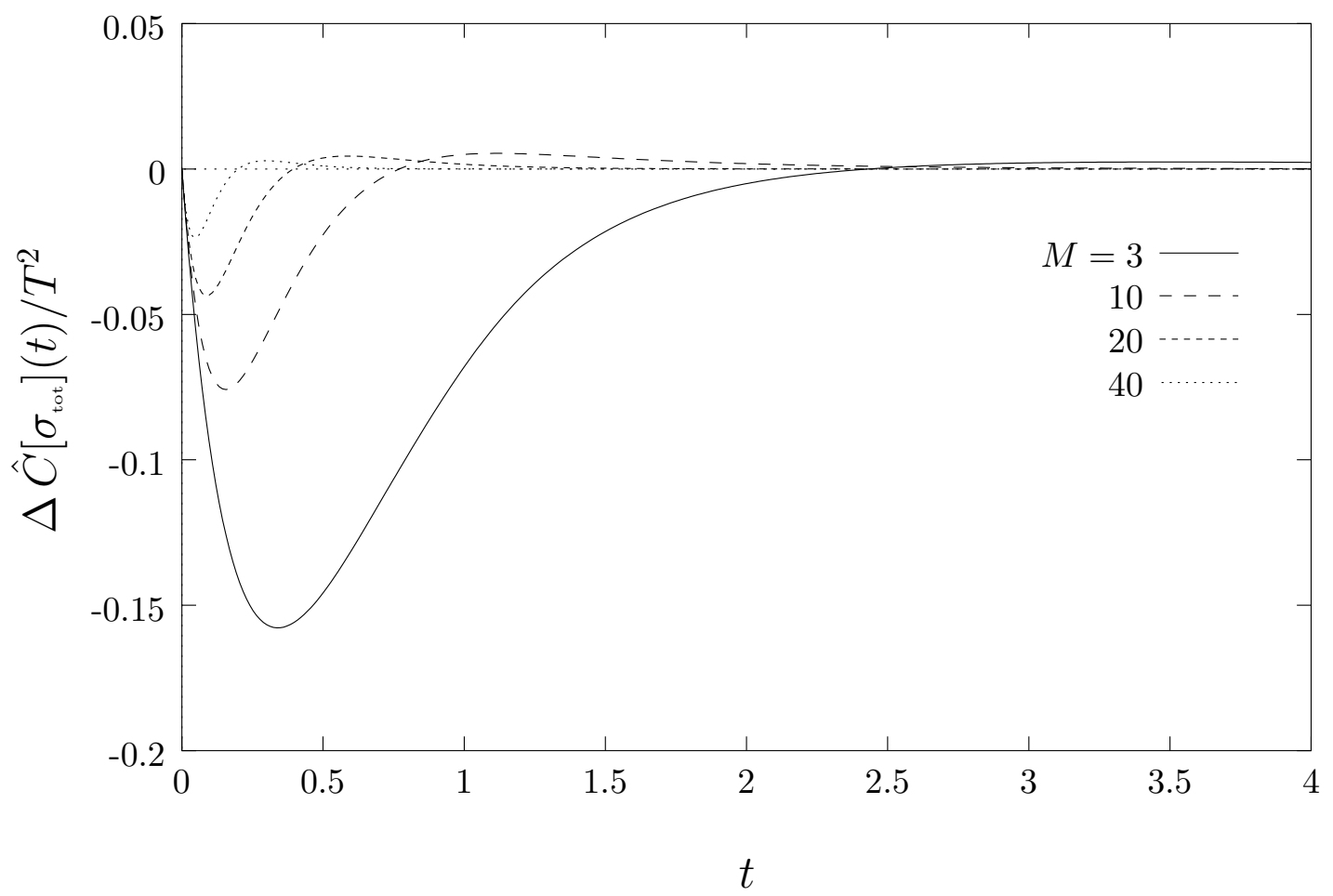

Figure 3: Difference between the rescaled Breit-Wigner approximation (27) and the exact result (36) for the autocorrelation function $\hat{C}\left[\sigma_{\text {tot }}\right](t)$ divided by $T^{2}$. The coupling strength is kept fixed $\kappa=0.1$, and the number of channels is varied: $M=3$ (solid line), $M=10$ (long dashed line), $M=20$ (short dashed line), and $M=40$ (dotted line).

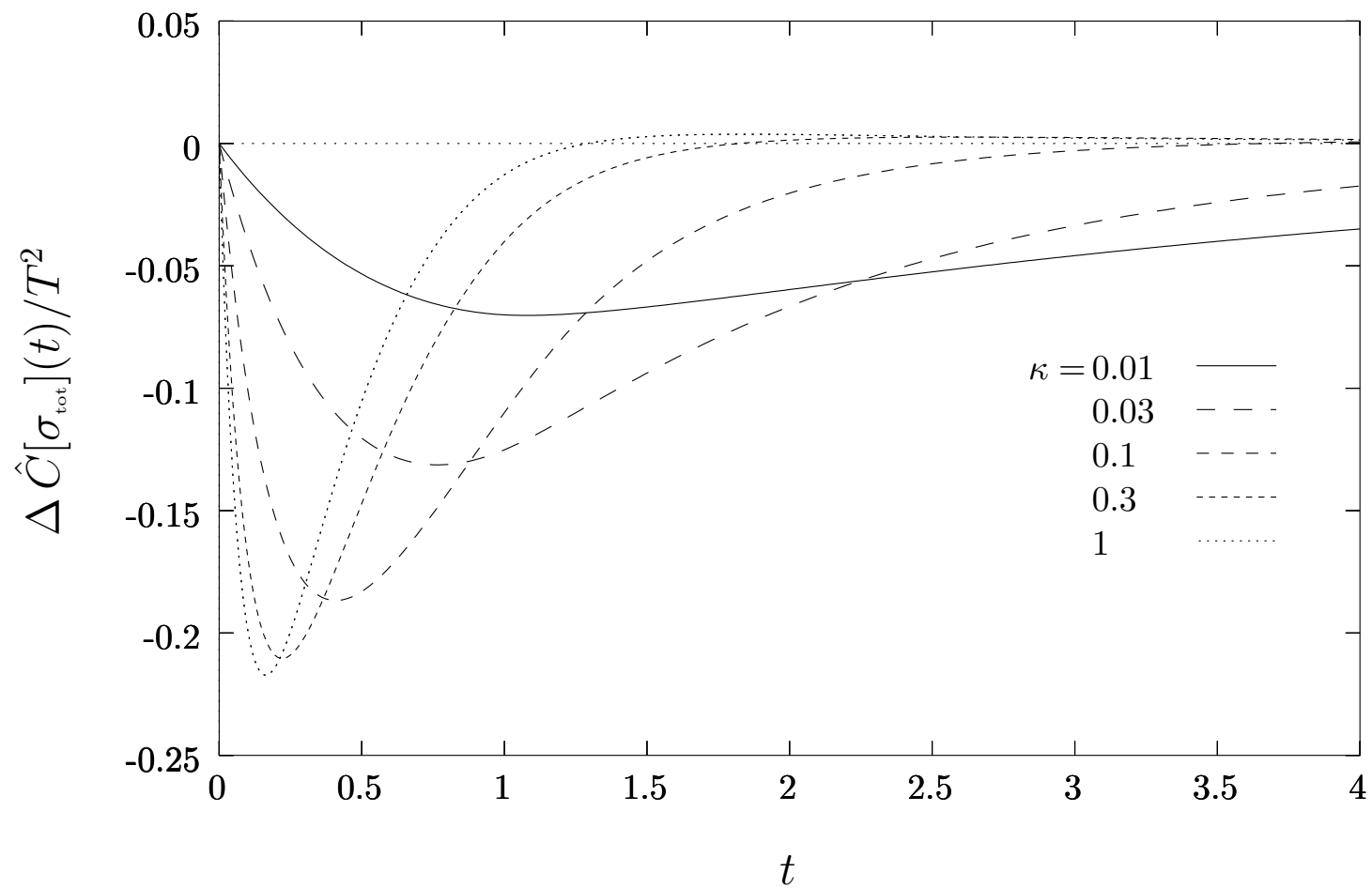

Figure 4: As in Fig. 3, but here, the number of channels $M=2$ is kept fixed, and the coupling strength is varied: $\kappa=0.01$ (solid line), $\kappa=0.03$ (long dashed line), $\kappa=0.1$ (medium dashed line), $\kappa=0.3$ (short dashed line), and $\kappa=1$ (dotted line). 


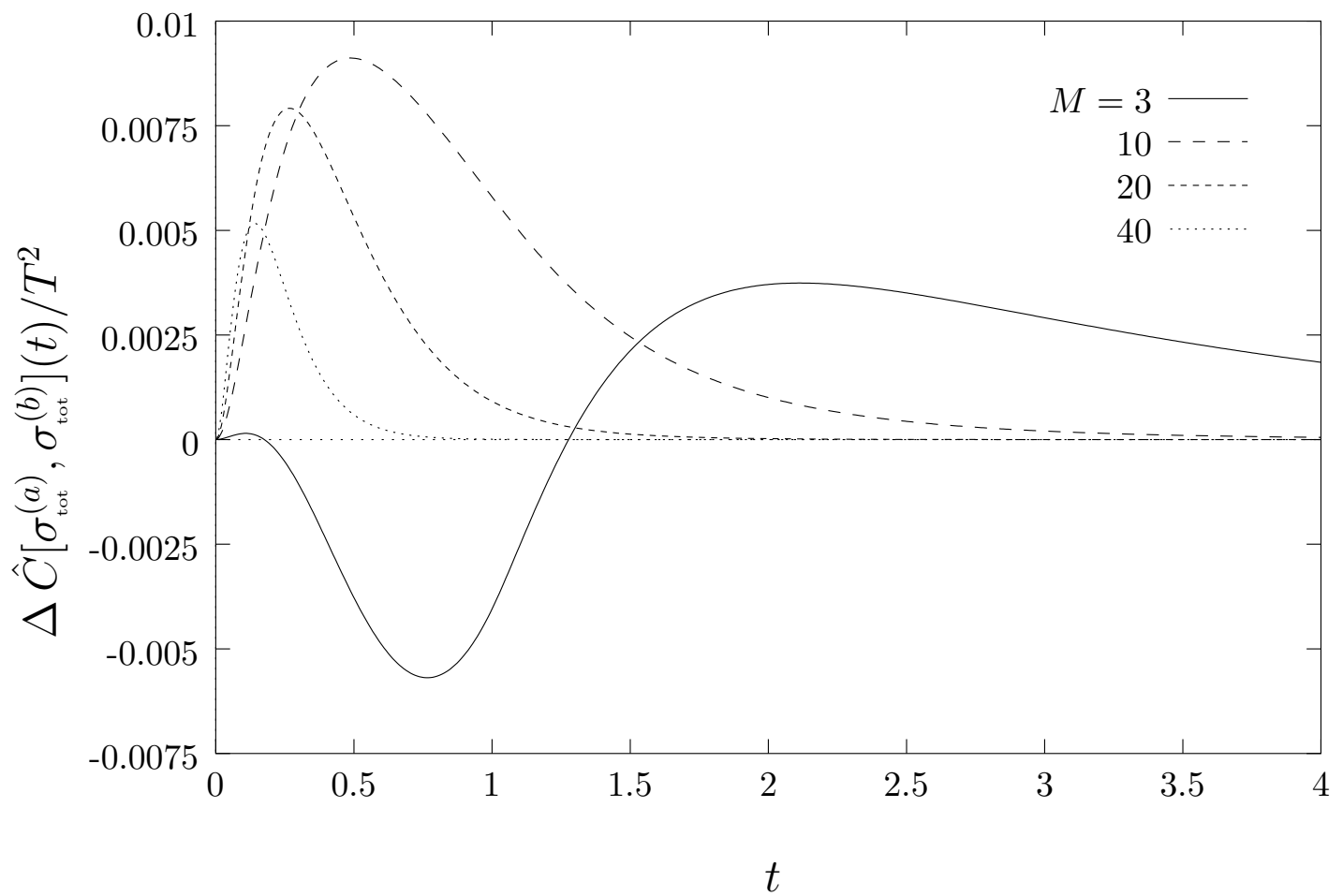

Figure 5: As in Fig. 3, but for the cross correlation function $\hat{C}\left[\sigma_{\text {tot }}^{a}, \sigma_{\text {tot }}^{b}\right](t)$ divided by $T^{2}$.

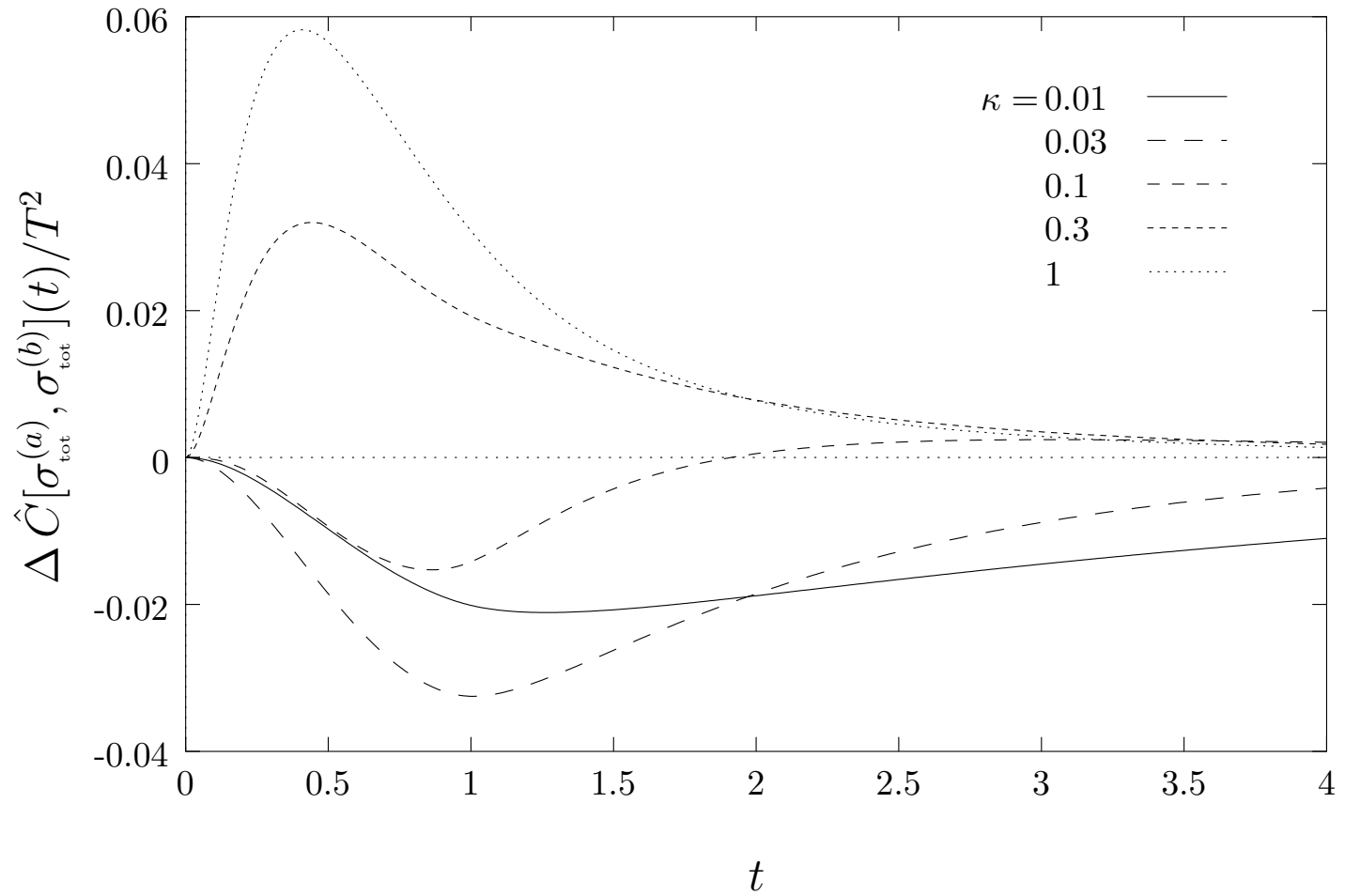

Figure 6: As in Fig. 团, but for the cross correlation function $\hat{C}\left[\sigma_{\text {tot }}^{a}, \sigma_{\text {tot }}^{b}\right](t)$ divided by $T^{2}$. 
of total cross sections, in the POE case. In this section we use the C-functions of total cross sections in the GOE case (for which we have exact analytic results) to check the validity of the rescaled Breit-Wigner approximation.

The Figs. 3 6 6 show the difference between the rescaled Breit-Wigner approximation (27) and the exact result (36) for C-functions of the total cross section in the GOE case. This is done for different numbers of channels (Fig. 3 and Fig. 5), and for different coupling strengths (Fig. 1 and Fig. 6). As the error of the rescaled BreitWigner approximation is zero for $t=0$ (see Sec. 5.1), all curves for the auto and cross C-functions begin at zero. In the case of the auto C-function the rescaled Breit-Wigner approximation mostly underestimates the exact result. In particular at small times, we get maximal deviations, which decrease again as $t \rightarrow \infty$. In the case of cross C-functions the error curves depend in a more irregular way on the number of channels or on the coupling strength. Though the error is of comparable size in all cases, the error curves at $t \rightarrow 0$ seem to behave linearly in the case of the auto C-functions and quadratically in the case of the cross C-functions (in agreement with our theoretical expectations; see Sec. 5.1). Yet the cross C-function vanishes for $\kappa=1$, while the approximation does not and must, therefore, be considered with great reservations. In general, the error is rather an absolute then a relative one. We may say that the rescaled Breit-Wigner approximation works quite well up to $\kappa=0.1$, and that for larger $\kappa$ the approximation still behaves qualitatively well except for the cross C-function for $\kappa=1$.

In Fig. 7, we compare the rescaled and the ordinary Breit-Wigner approximation. The latter is obtained by undoing the rescaling and replacing $T$ by $4 \kappa$ in Eq. (27). As we have no exact theory in the POE case [Fig. 耳(b)], we use numerical Monte Carlo data instead ( $c f$. Sec. 7). The data may be considered as exact up to the small fluctuations, seen in the data points. Though the resonances are well separated for $\kappa=0.1$ which is used here, the pure Breit-Wigner approximation is already far off the exact result, in particular at small times. This is true for the GOE case shown in Fig. I (a), as well as for the POE case in Fig. If(b). In fact, the rescaled Breit-Wigner prediction in the GOE case is much closer to the POE case, which makes it practically impossible to distinguish between correlated and uncorrelated spectra. Note that the auto C-function shown here, is monotonously decreasing without showing any qualitative sign of the correlation hole.

The rescaled Breit-Wigner approximation by contrast, reproduces the exact values of the C-function at $t=0$ in the GOE and apparently also in the POE case. This allows to detect the correlation hole if present, at least in principle (absolute cross sections must be available with sufficient accuracy). Though the rescaled Breit-Wigner approximation underestimates the auto C-function at intermediate times in the GOE case [Fig. [ ( 1 (a)], it agrees very nicely with the numerical data in the POE case [Fig. 团(b)].

It seems that the rescaled Breit-Wigner accounts very well for uncorrelated resonances that diffuse independently into the complex plane (similar to what one would expect from an effective mean field theory). For resonances which are strongly correlated from the very beginning, the dynamics of the resonances is more complicated, and the rescaling procedure cannot fully account for that. However, in cases where deviations of a few percents (of the maximal C-function value) are still acceptable, the rescaled Breit-Wigner approximation can be applied up to quite large coupling strengths, which reach well into the strong absorption regime. While it is convenient to consider the absolute error at small times (in the correlation hole region), the relative error is more appropriate at large times, i.e. in the tails of the C-function. The latter apparently scales with the total transmission $\sum_{a=1}^{M} T_{a}$ (we have checked this separately), in line with earlier theoretical work [34].

\section{Numerical analysis: GOE versus POE}

Here we perform Monte Carlo simulations for correlation functions of partial and total cross sections. The aim is to distinguish the situations, where the internal Hamiltonian has a purely random spectrum (POE), from those where it has a spectrum with correlations (GOE). We restrict the analysis to coupling strengths $0<\kappa \leq 1$ that covers the whole range for the transmission coefficient. As discussed amongst others in Refs. [7, 39, 40 the scattering ensemble may show quite a different behavior when $\kappa \gg 1$. The results are presented for two open channels. Calculations for larger channel numbers have been performed, but they show no significantly different behavior. In the case of total cross sections and GOE, this can be seen from the results for the VWZ integral obtained in Sec. V (see for example Fig. 1). In the other cases, i.e. POE or partial cross sections, the rescaled Breit-Wigner approximation gives the same answer [see Eq. (31)]. In all cases the number of channels merely enters as a scale factor.

The numerical data are obtained using Eq. (9), i.e. we calculate the Fourier transforms of the two cross sections in question and then take the ensemble average (400 runs) over their (complex conjugated) product. Finally the product of the average cross sections is subtracted to eliminate the peak at $t=0$. The dimension of the effective Hamiltonian $H$ [see Eq. (11)] is $N=300$. Where the resulting data are smoothed, this is done using 


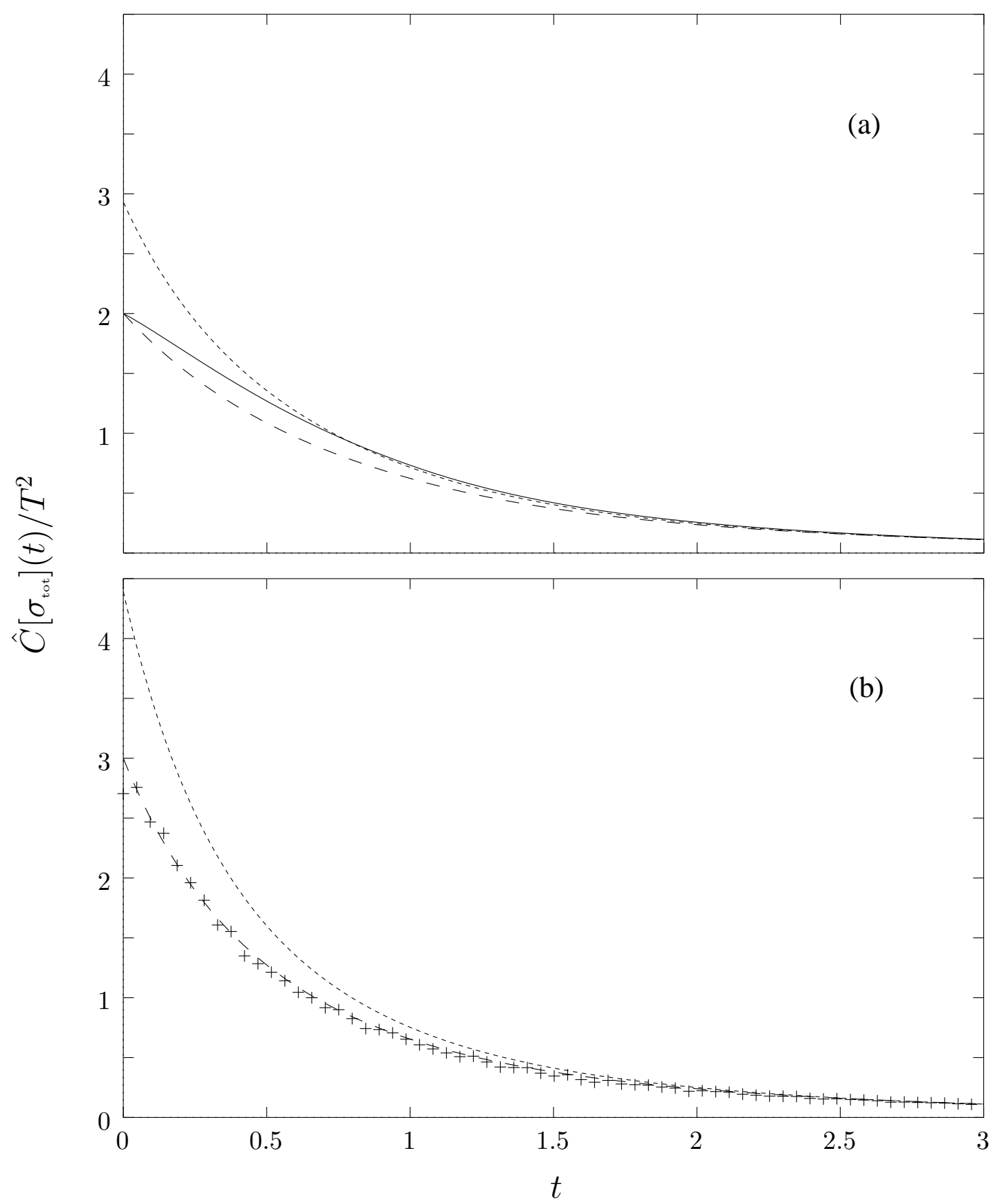

Figure 7: The autocorrelation function $\hat{C}\left[\sigma_{\text {tot }}\right](t)$ divided by $T^{2}$ for $M=2$ and $\kappa=0.1$. The long dashed line is the rescaled Breit-Wigner approximation (27), and the short dashed line is the ordinary Breit-Wigner approximation (details see text). In (a) we show the GOE and in (b) the POE case. The solid line in (a) shows the exact result (36), whereas the crosses in (b) show numerical Monte Carlo calculations. 


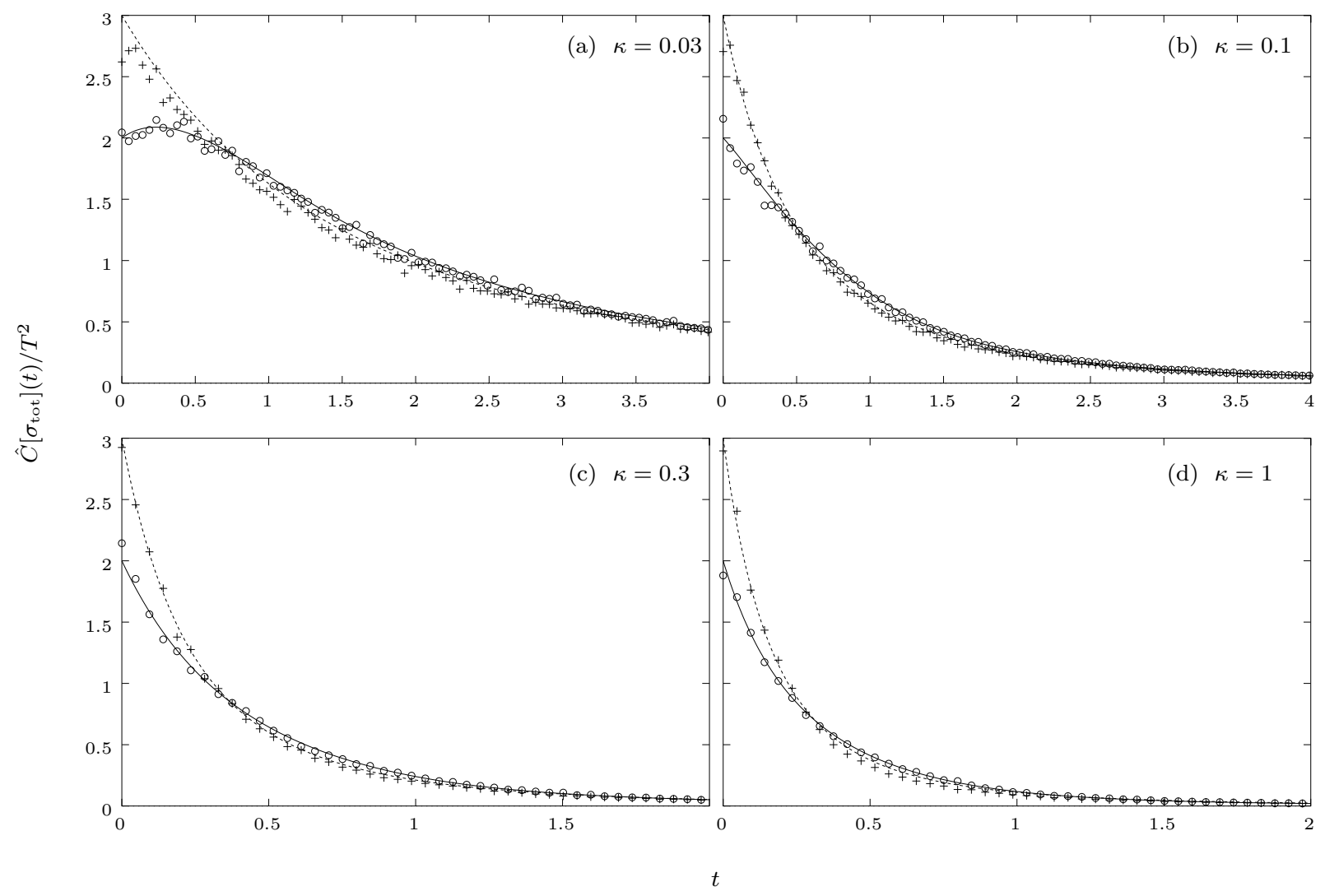

Figure 8: The autocorrelation function of the total cross section divided by $T^{2}$. The GOE versus the POE case, for coupling strengths: $\kappa=0.03$ (a), $\kappa=0.1$ (b), $\kappa=0.3$ (c), and $\kappa=1$. (d). The circles show the smoothed numerical GOE data; the crosses the equally treated POE data. In all cases (a), . , (d) the exact VWZ integral is used for the GOE theory (solid line) and the rescaled Breit-Wigner approximation for the POE (dashed line).

a $\chi^{2}$-fit to a natural cubic spline. The length of its curve segments was 28 points that corresponds to $\Delta t \approx 0.26$ 41. From the spline, 150 equidistant points in $t$ are finally plotted. Note, that there is no free fit parameter, and the curves are not normalized. The factor $T^{2}$ by which we divide, is directly determined from the input parameter $\kappa$, using Eq. (6).

\subsection{Total cross sections}

We start the numerical analysis with auto and cross correlation functions of total cross sections. While this case is relatively easy to handle in theory, it is usually very difficult to obtain total cross sections from an experiment, in particular if two independent total cross sections are needed.

\section{Autocorrelation functions}

As a starting point consider the correlation hole [2] in the Fourier transform of a discrete energy spectrum $\hat{C}(t) \sim 1-b_{2}(t)$, that distinguishes the GOE from a purely random spectrum (POE). In the limit of vanishing coupling $\kappa \rightarrow 0$, the auto C-function $\hat{C}\left[\sigma_{\text {tot }}\right](t)$ divided by $T^{2}$ converges not to $\hat{C}(t)$, but to $\hat{C}(t)+2$. The reason is that the resonances are weighted by Porter-Thomas distributed intensities (or partial widths) [ [ \&], which leads to a reduced correlation hole of $1 / 3$.

Figure 8 shows $\hat{C}\left[\sigma_{\text {tot }}\right](t) / T^{2}$, for the GOE and the POE case. We find the behavior described above, but with the difference, that due to the finite coupling $\hat{C}(t)$ must be multiplied with roughly an exponential decaying function. In the four panels (a)-(d), the coupling parameter is increased from $\kappa=0.03$ to $\kappa=1$. In the GOE case the numerical results are compared with the exact theory, Eq. (36), but in the POE case we are dependent on the rescaled Breit-Wigner approximation, Eq. (27). We find perfect agreement for the GOE which shows that the numerical Monte-Carlo calculation is reliable. Interestingly the rescaled Breit-Wigner approximation describes the POE data almost as well. Only for $\kappa=1$ we find minor deviations at intermediate times. This reaffirms, that the rescaled Breit-Wigner approximation is best suited for cases where the correlations are relatively weak. 


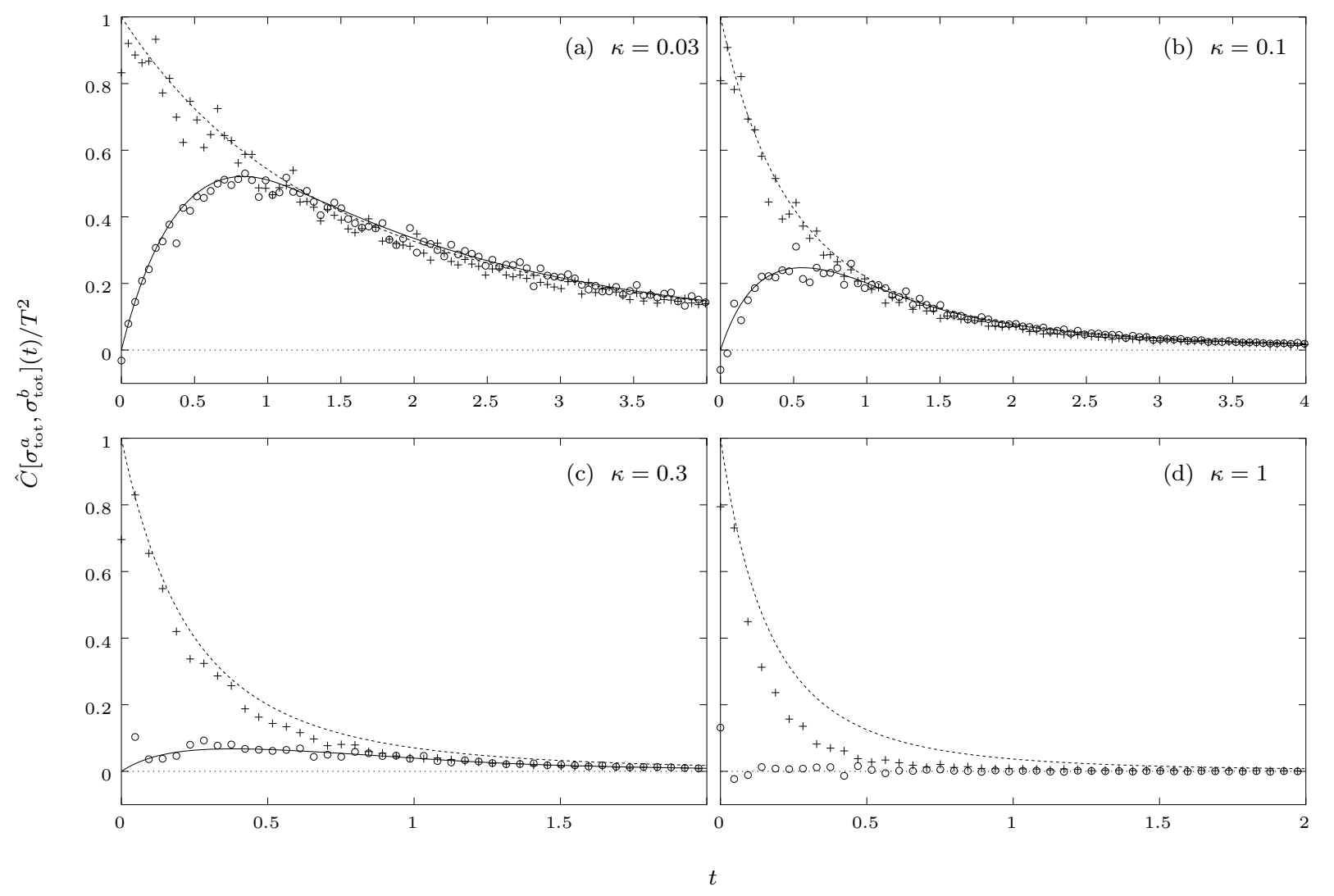

Figure 9: As in Fig. 8, but for the cross correlation function divided by $T^{2}$. Note, however, that in part (d), the C-function in the GOE case is exactly zero, and not plotted.

\section{Cross correlation functions}

Let us again think of a discrete stick spectrum where the levels are weighted by intensities (or partial widths). In order to calculate the C-function between two spectra related to different channels, it is reasonable to assume that the positions of the resonances are the same, but the corresponding intensities are uncorrelated. Then this cross C-function becomes: $\hat{C}(t) \sim 1-b_{2}(t)$, i.e. we obtain the full correlation hole [42] (see also the Eqs. (23)-(27) and the discussion following them). For the GOE case, this has been shown numerically in Sec. 5.2.

In Fig. 9 we show numerical and theoretical results for the cross C-function. In the GOE case we plot the numerical results together with the exact theory, Eq. (36), and we find again perfect agreement well within the statistical error (note that for $\kappa=1$, the cross correlation function vanishes identically). In the POE case we compare our numerical data with the rescaled Breit-Wigner approximation, Eq. (27). In the panels (a) and (b), which correspond to the weak coupling case, the theory agrees very well with the numerical data. For $\kappa=0.3(\mathrm{c})$, however, we find first systematic deviations. The rescaled Breit-Wigner approximation begins to overestimate the true C-function, which becomes even worse for $\kappa=1$ (d).

Possibly the deviations for $\kappa \gtrsim 0.3$ may be due to the appearance of correlations when the resonances begin to overlap. The fact that the deviations are more pronounced here, than in the case of auto C-functions can be explained in a natural way by the higher sensitivity of the cross C-function to correlations. Moreover, it has been proven in Ref [18] (though only for the one channel case) that the coupling to decay channels may indeed induce correlations even in the regime $\kappa<1$.

In the GOE case, we know from Sec. 5.1 that the rescaled Breit-Wigner approximation reproduces the exact value of auto and cross C-functions at $t=0$ for arbitrary coupling strengths. This can also be proved for the POE in the one channel case 43 . Here, Fig. 8 and Fig. 9 give numerical evidence, that the same is true in the many channel case as well. Thus the relative size of the correlation hole seems to be independent of the coupling strengths, which turns the correlation hole into a persisting signature of chaos even in the limit of strong transmission $\forall a: T_{a} \rightarrow 1$. This may be contrasted to the behavior in the energy domain, where the correlation hole for $C\left[\sigma_{\text {tot }}^{(a)}, \sigma_{\text {tot }}^{(b)}\right](\omega)$ gradually disappears with increasing number of channels or total transmission. Note, 


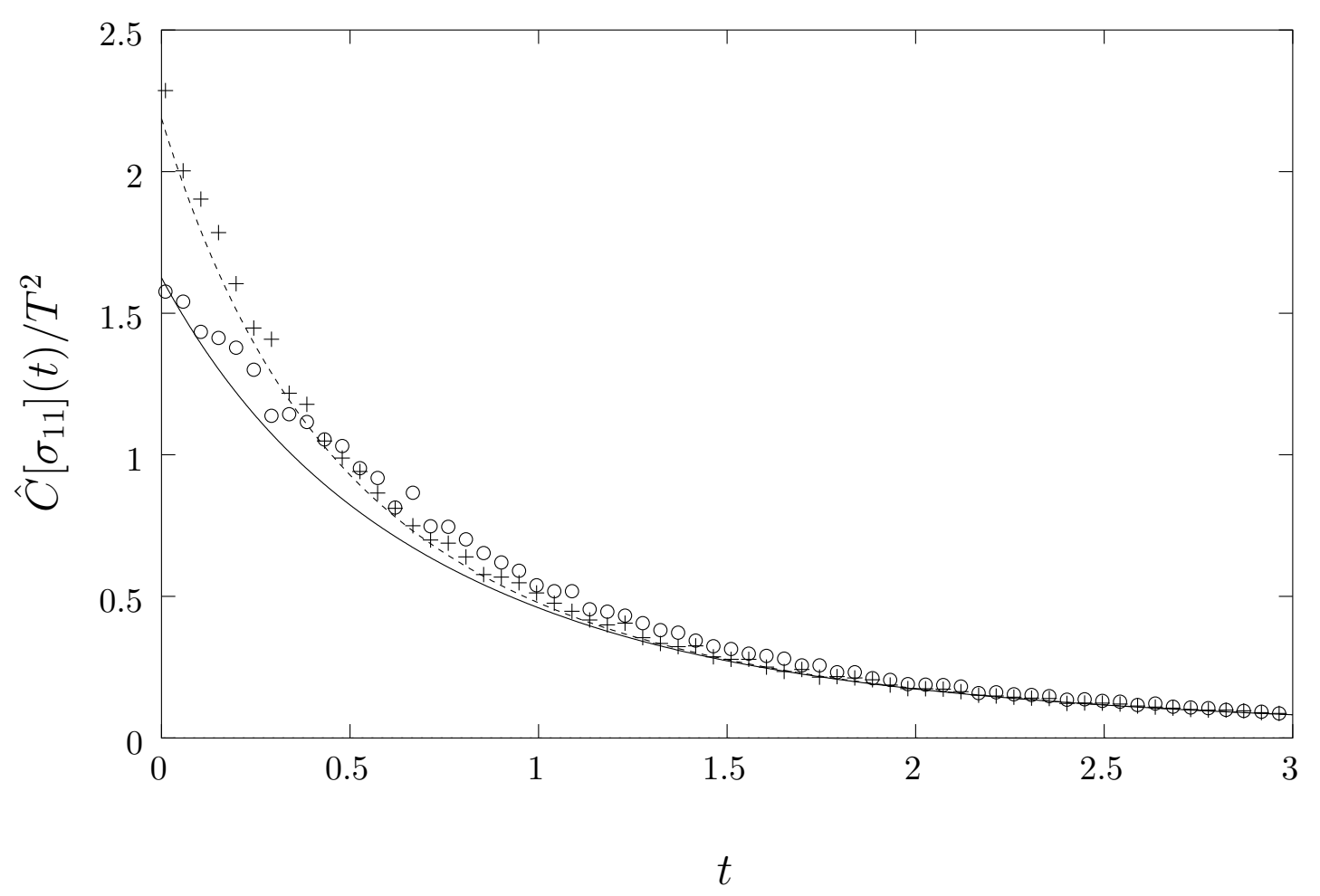

Figure 10: The autocorrelation function for the elastic cross section divided by $T^{2}$ with the coupling strength $\kappa=0.1$. The numerical data are shown by circles (GOE) and crosses (POE). The theoretical curves are the rescaled Breit-Wigner approximation, Eq. (31), for the GOE (solid line) and the POE (dashed line).

however, that in the time domain, the correlation hole becomes compressed to short times. One would then need very long spectra with many resonances to resolve the correlation hole. The generality of this invariance property and its implications will be the subject of future studies.

\subsection{Partial cross sections}

In this section we show results of Monte Carlo calculations for C-functions of partial cross sections. Though we obtained a closed formula even here (using the diagonal approximation for the partial cross sections and the rescaled Breit-Wigner approximation for the correlation function), we must be prepared for the approximation to deviate relatively early from the true result. The main advantage of partial cross sections, is their easy experimental accessibility. According to our approximation, the relative size of the correlation hole is given by:

$$
Q=\frac{B}{A} \frac{(1+6 / M)(1+4 / M)}{(1+2 / M)^{2}}
$$

(see Eq. (31) and the list below). Note that for particular choices of the partial cross sections, this quotient may become even larger than one, which implies that the corresponding $\mathrm{C}$-function for the GOE becomes negative at small times.

\section{Autocorrelation functions}

According to Eq. (31) we have to distinguish between the auto C-function of an elastic or inelastic cross section. In the elastic case, $B / A=3 / 35$ so that the relative size $Q$ of the correlation hole varies from $Q=3 / 35 \approx 0.0857$ for $M \rightarrow \infty$, and $9 / 35 \approx 0.257$ for $M=2$. In the inelastic case, $B / A=1 / 9$ so that $Q$ varies from $Q=1 / 9 \approx 0.111$ for $M \rightarrow \infty$, and $Q=1 / 3 \approx 0.333$ for $M=2$.

In Fig. 10 (elastic case) and Fig. 11 (inelastic case) we set the coupling parameter to $\kappa=0.1$. The numerical data is shown together with our approximate result, Eq. (31). In both figures, the approximation clearly deviates from the numerical result (which we may consider to be exact). The deviations are more pronounced in the GOE case, which is in line with earlier observations in the context of total cross sections. The deviations occur just in the region of the correlation hole, and thus diminish the difference between GOE and POE considerably. 


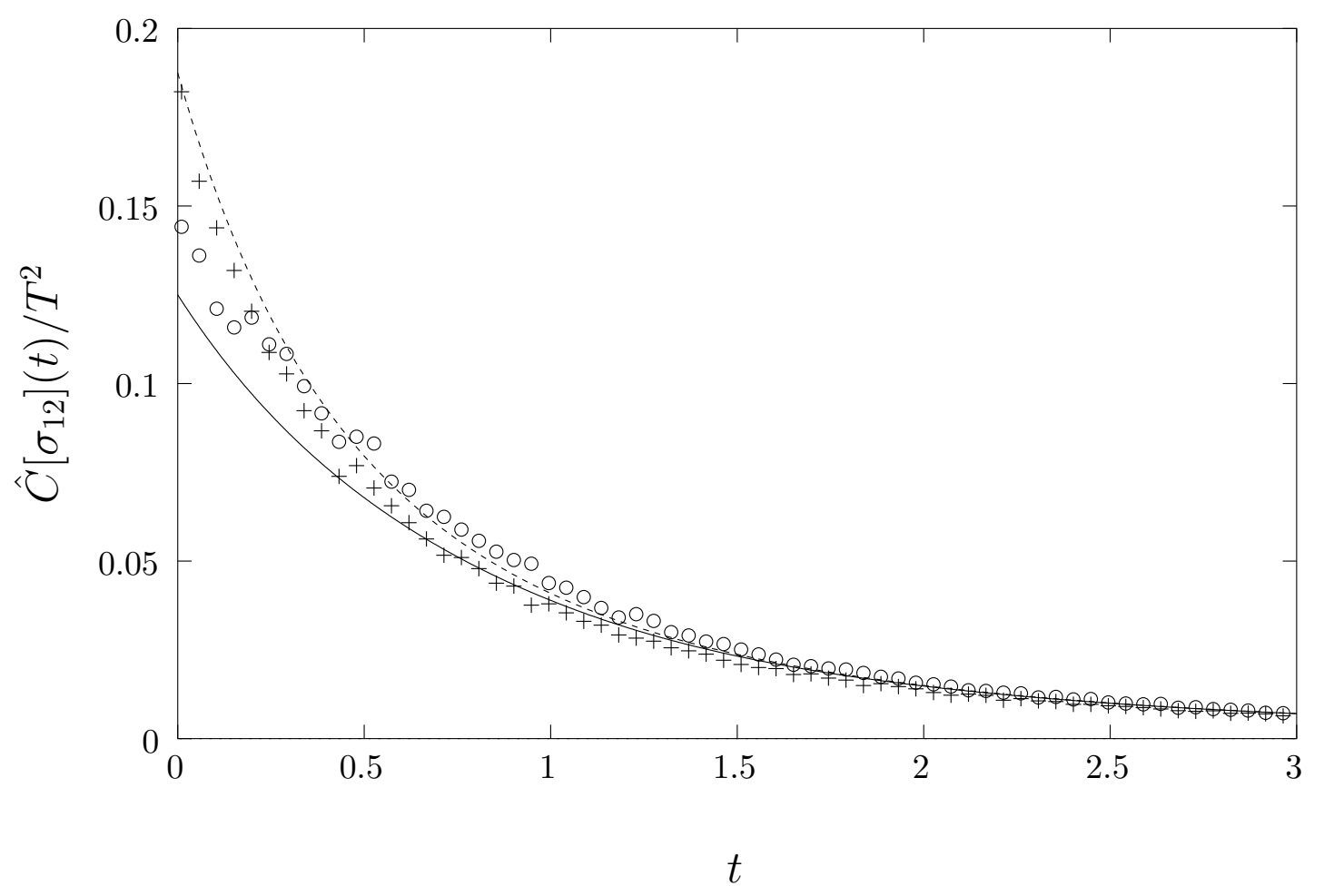

Figure 11: As Fig. 10, but for inelastic cross sections.

In Fig. 10 it seems, that the theoretical values at $t=0$ are still quite accurate, so that the correlation hole could be detected there. However, the curves for the GOE and POE merge very rapidly (at $t \approx 0.5)$. In Fig. 11 we see that our approximation fails even at $t=0$. The numerical curves for POE and GOE lie so close together, that it is practically impossible to identify the correlation hole. Similar investigations 8 gave essentially the same results.

\section{Cross correlation functions}

As the number of channels is $M=2$, we need to consider only two different types of cross C-functions: the C-function of two elastic cross sections, and of an elastic and an inelastic cross section (sharing the same entrance channel). In the list below Eq. (31) we find that $B / A=1$ in the first case, and $B / A=1 / 5$ in the latter. Hence we may expect to obtain considerably larger correlation holes. For the case of two elastic cross sections the relative size $Q$ of the correlation hole is $Q=1$ for $M \rightarrow \infty$ and $Q=3$ for $M=2$. This means, that the C-function in the GOE case becomes negative at small times. For the case of one elastic and one inelastic cross section, $Q=1 / 5$ for $M \rightarrow \infty$ and $Q=3 / 5$ for $M=2$. This is the most natural constellation in experiments. Unfortunately the corresponding correlation hole is not very large.

Figure 12 shows the cross C-function of two elastic cross sections divided by $T^{2}$. In the four panels (a)-(d) the coupling strength is increased from $\kappa=0.03$ up to $\kappa=1$. The correlation hole is so big $(Q=3)$, that the GOE curve becomes indeed negative at small $t$, with the only exception of panel (d). The rescaled Breit-Wigner approximation works quite well in (a) and (b), begins to fail in (c), and fails completely in (d). Surprisingly the error becomes large in the POE case first, while the agreement in the GOE case is still reasonable. This contradicts to some extent, what we have found and partially explained in the case of total cross sections. Further studies are probably necessary to clarify this point.

Figure 13 shows the cross C-function of an elastic and an inelastic cross section. Not only is the correlation hole smaller, the deviations between our approximation and the numerical data are also larger than in the previous case (Fig. 12). In panel (b) for $\kappa=0.1$ we find already noticeable deviations in the POE case, and the correlation hole has practically disappeared for $\kappa=0.3$, in panel (c). In panel (d) finally, the numerical data practically coincide for the POE and the GOE case. It seems that the C-function in this case is negative for any value of $t$. Again this is an aspect, which deserves further studies.

In this subsection, we saw that the validity of our approximation for partial cross sections is restricted to 


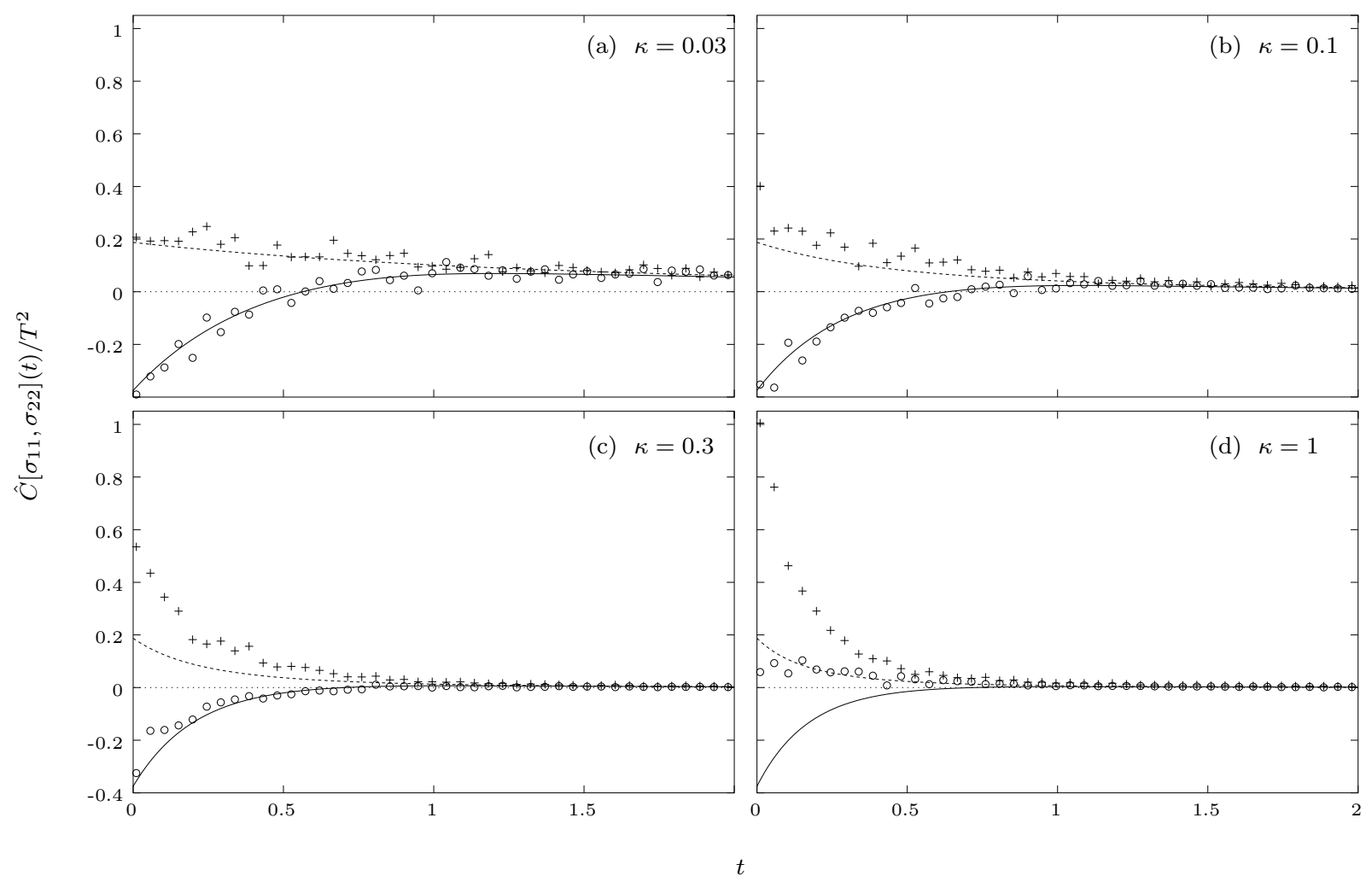

Figure 12: The cross correlation function $\hat{C}\left[\sigma_{11}, \sigma_{22}\right](t)$ divided by $T^{2}$. The coupling strength is varied: $\kappa=0.03$ (a), $\kappa=0.1(\mathrm{~b}), \kappa=0.3$ (c), and $\kappa=1$ (d). The numerical data is shown with circles (GOE) and crosses (POE). The rescaled Breit-Wigner approximation (31) is plotted for the GOE (solid line) and for the POE (dashed line).

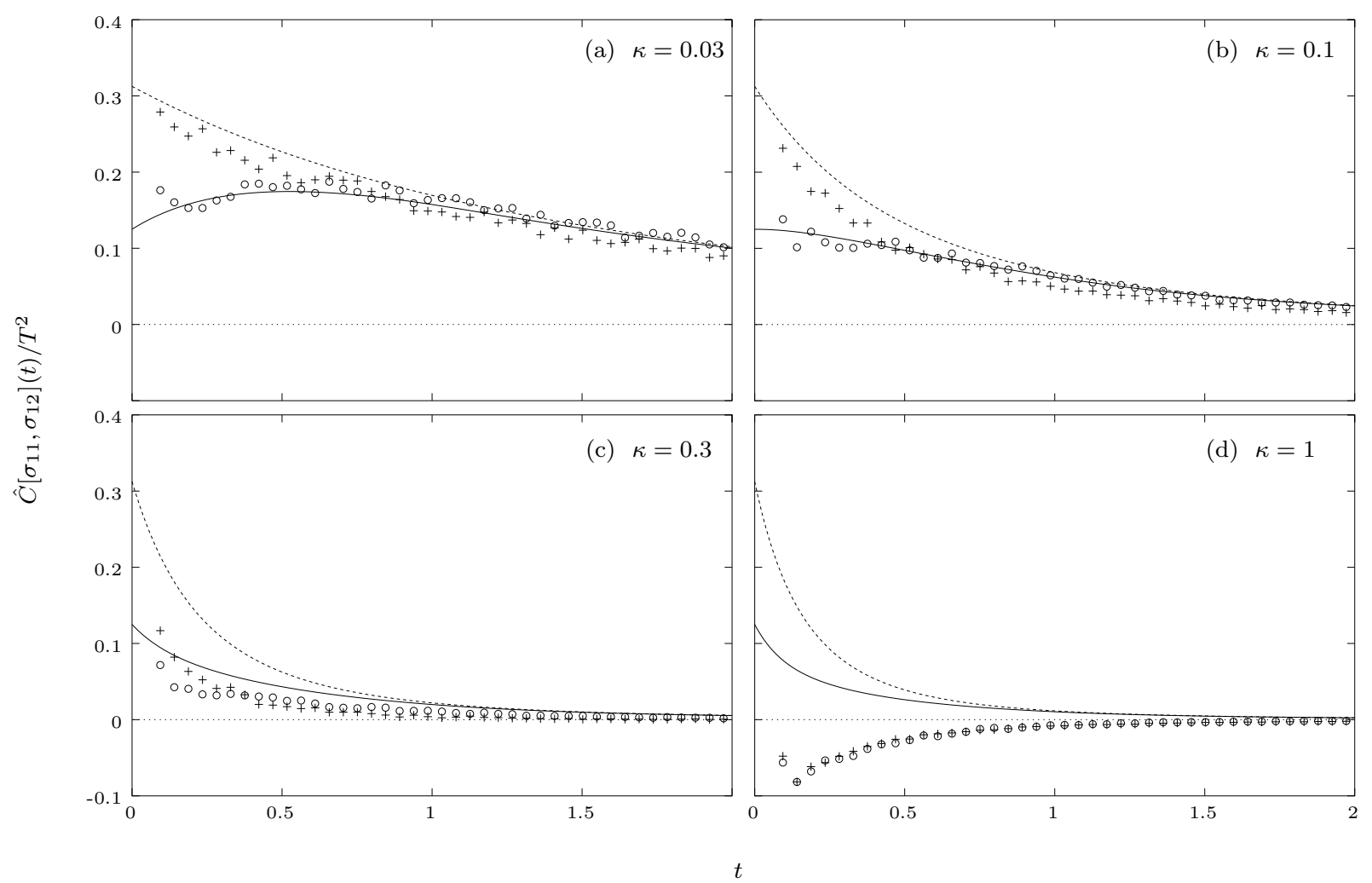

Figure 13: As in Fig. 12, but for the cross correlation function between an elastic and an inelastic cross section $\hat{C}\left[\sigma_{11}, \sigma_{12}\right](t)$ divided by $T^{2}$. 
coupling strengths that are typically much smaller than in the case of total cross sections. Moreover, we can no longer obtain the exact value of the $\mathrm{C}$-functions at $t=0$. However, we found here the largest effects of the correlation hole. In particular, the case of two elastic cross sections is very promising in view of a possible realization in an experiment.

\section{Summary}

By introducing an appropriate rescaling of the coupling strengths in the conventional theory of multichannel scattering for weak absorption, we have been able to extend its domain of validity far into the region of overlapping resonances. We have used this method (which we call the rescaled Breit-Wigner approximation) to calculate auto and cross correlation functions for total and partial cross sections both for the POE and the GOE case, i.e. in situations we consider as "integrable" and "chaotic."

Starting from the VWZ integral [9], which describes the GOE case, we have performed exact calculations for the correlation functions of total cross sections in the time domain. By comparison with these results and numerics, we determined the domain of validity of the rescaled Breit-Wigner approximation. It turned out, that it works particularly well in the case of weak correlations.

We showed the differences between POE and GOE all the way up to the domain of strong transmission in all channels, and we found that autocorrelation functions are a poor means of determining the chaoticity or integrability of a scattering system in strong absorption, whereas certain cross correlation functions are quite sensitive. A particularly strong effect is seen in cross correlation functions of two different total cross sections. Unfortunately total cross sections are usually very hard to measure and, therefore, this observation may be of little practical value. As far as partial cross sections are concerned, we find that cross correlations of two elastic cross sections are also very sensitive, far more than either the ones between an elastic and an inelastic cross section or between two inelastic ones.

For systems where two asymptotic channels are stable the cross correlation of two elastic cross sections is a very attractive means of analyzing the reaction. Also at least for intermediate coupling, i.e. overlapping resonances with moderate coupling in each channel we have a theoretical result with which to compare. This is particularly important for the integrable case where we know that nongeneric behavior might be quite common. First experiments on microwave billiards to check our results are on their way.

\section{Acknowledgments}

This work was supported by CONACyT grant 25192-E, and by DGAPA (UNAM) grant IN-109000. We thank M. Müller for numerical calculations, and M. Müller and I. Rotter for discussions in particular in the early stage of this work, as well as Y. Alhassid for usefull comments.

\section{Average S-matrix}

The S-matrix given in Eq. (11) may equivalently be expressed in terms of the so called "K-matrix" 14]:

$$
S=\frac{1-\mathrm{i} K}{1+\mathrm{i} K}, \quad K(E)=\frac{1}{2} V^{\dagger} \frac{1}{E-H_{\mathrm{int}}} V .
$$

As we will see below, the average S-matrix can be obtained easily from the average K-matrix. This offers a convenient way to define the openness of the system, i.e. the coupling strengths to the decay channels.

In the eigenbasis of $H_{\mathrm{int}}$ we get the following expression for the elements of the K-matrix (61):

$$
K_{a b}(E)=\frac{1}{2} \sum_{i=1}^{N} \frac{V_{i a} V_{i b}}{E-\varepsilon_{i}},
$$

where $\left\{\varepsilon_{i}\right\}$ are the eigenvalues of the closed system $H_{\mathrm{int}}$. The coupling matrix elements $\left\{V_{i a}\right\}$ and the eigenvalues $\left\{\varepsilon_{i}\right\}$ are statistically independent in the ensembles considered (the GOE and the POE). Therefore, we get for the average of $K_{a b}(E)$ :

$$
\left\langle K_{a b}\left(E^{+}\right)\right\rangle=\delta_{a b} \frac{N}{2}\left\langle V_{i a} V_{i b}\right\rangle\left\langle\frac{1}{E-\varepsilon+\mathrm{i} 0}\right\rangle_{\rho},
$$


which holds for any $i$. Here $\langle\ldots\rangle_{\rho}$ stands for the average over the level density $\rho$. Using that $\left\langle V_{i a} V_{j b}\right\rangle=$ $\delta_{i j} \delta_{a b}\left\langle V_{i a}^{2}\right\rangle$, which again holds for the scattering ensembles considered here (see Sec. 2), we get:

$$
\left\langle K_{a b}\left(E^{+}\right)\right\rangle=\delta_{a b} \frac{N}{2}\left\langle V_{i a}^{2}\right\rangle\left[f \mathrm{~d} \varepsilon \frac{\rho(\varepsilon)}{E-\varepsilon}-\mathrm{i} \pi \rho(E)\right],
$$

where we split the integral used to average over the level density, into its principle value part and the residue. Note, that the average K-matrix is diagonal. We define the center $E_{0}=0$ of the spectrum as that point where the principle value integral vanishes. Then we obtain:

$$
\left\langle K_{a a}\left(0^{+}\right)\right\rangle=-\frac{\mathrm{i} \pi}{2} N\left\langle V_{i a}^{2}\right\rangle \rho(0) .
$$

Finally we define the "coupling parameters" $\kappa_{a}$ as follows:

$$
\kappa_{a}=\mathrm{i}\left\langle K_{a a}\left(0^{+}\right)\right\rangle=\frac{\pi}{2 d}\left\langle V_{i a}^{2}\right\rangle, \quad d=\frac{1}{N \rho(0)} .
$$

It can be shown 22] that due to the analytic properties of the S-matrix its average is directly related to the average K-matrix:

$$
\langle S(E)\rangle=\frac{1-\mathrm{i}\left\langle K\left(E^{+}\right)\right\rangle}{1+\mathrm{i}\left\langle K\left(E^{+}\right)\right\rangle} .
$$

The average S-matrix is also diagonal. In the center of the spectrum the average K-matrix is purely imaginary, so that $\langle S(E)\rangle$ is real. Its elements are then given by:

$$
\left\langle S_{a a}(0)\right\rangle=\frac{1-\kappa_{a}}{1+\kappa_{a}} .
$$

\section{References}

[1] E. P. Wigner and C. Eisenbud, Phys. Rev. 72, 29 (1947).

[2] L. Leviandier, M. Lombardi, R. Jost, and J. P. Pique, Phys. Rev. Lett. 56, 2449 (1986).

[3] T. Guhr and H. A. Weidenmüller, Chem. Phys. 146, 21 (1990).

[4] M. Lombardi and T. H. Seligman, Phys. Rev. A 47, 3571 (1993).

[5] C. Jung and T. H. Seligman, Phys. Rep. 285, 77 (1997).

[6] M. V. Berry and M. Tabor, Proc. R. Soc. London, Ser. A 356, 375 (1977).

[7] F.-M. Dittes, I. Rotter, and T. H. Seligman, Phys. Lett. A 158, 14 (1991).

[8] H. Alt, H.-D. Gräf, T. Guhr, H. L. Harney, R. Hoffbert, H. Rehfeld, A. Richter, and P. Schardt, Phys. Rev. E 55, 6674 (1997).

[9] J. J. M. Verbaarschot, H. A. Weidenmüller, and M. R. Zirnbauer, Phys. Rep. 129, 367 (1985).

[10] G. Breit and E. Wigner, Phys. Rev. 49, 519 (1936).

[11] G. R. Satchler, Phys. Lett. 7, 55 (1963).

[12] H. Feshbach, Ann. Phys. (NY) 5, 357 (1958).

[13] H. Feshbach, Ann. Phys. (NY) 19, 287 (1962).

[14] C. Mahaux and H. A. Weidenmüller, Shell-Model Approach to Nuclear Reactions (North-Holland, Amsterdam, 1969).

[15] P. Kleinwächter and I. Rotter, Phys. Rev. C 32, 1742 (1985).

[16] C. A. Engelbrecht and H. A. Weidenmüller, Phys. Rev. C 8, 859 (1973). 
[17] M. L. Mehta, Random Matrices and the Statistical Theory of Energy Levels, (Academic Press, Boston, 1991).

[18] T. Gorin, J. Phys. A 32, 2315 (1999).

[19] A. Pandey, Ann. Phys. (N. Y.) 119, 170 (1979).

[20] I. H. Chan, R. M. Clarke, C. M. Marcus, K. Campman, and A. C. Gossard, Phys. Rev. Lett. 74, 3876 (1995).

[21] E. Oran Brigham, The Fast Fourier Transform, (Prentice-Hall, Englewood Cliffs, N. Y., 1974).

[22] P. A. Mello, P. Pereyra, and T. H. Seligman, Ann. Phys. (NY) 161, 254 (1985).

[23] Y. V. Fyodorov and H.-J. Sommers, J. Math. Phys. 38, 1918 (1997).

[24] H. L. Harney, F.-M. Dittes, and A. Müller, Ann. Phys. (NY) 220, 159 (1992).

[25] T. Gorin, PhD thesis, Technische Universität Dresden, 1998.

[26] W. Hauser and H. Feshbach, Phys. Rev. 87, 366 (1952).

[27] A. Müller and H. L. Harney, Phys. Rev. C 35, 1228 (1987).

[28] D. Agassi, H. A. Weidenmüller, and G. Mantzouranis, Phys. Rep. 22, 145 (1975).

[29] A. Müller and H. L. Harney, Phys. Rev. C 37, 2435 (1988).

[30] T. Ericson and T. Mayer-Kuckuk, Annu. Rev. Nucl. Sci. 16, 183 (1966).

[31] C. E. Porter and R. G. Thomas, Phys. Rev. 104, 483 (1956).

[32] Y. Alhassid and Y. V. Fyodorov, J. Phys. Chem. A 102, 9577 (1998).

[33] Y. V. Fyodorov and Y. Alhassid, Phys. Rev. A 58, R3375 (1998).

[34] J. J. Verbaarschot, Ann. Phys. (N. Y.) 168, 368 (1986).

[35] H. L. Harney and A. Hüpper, Z. Phys. A 328, 327 (1987).

[36] H. L. Harney, A. Hüpper, M. Mayer, and A. Müller, Z. Phys. A 335, 293 (1990).

[37] A. Müller and H. L. Harney, Z. Phys. A 337, 465 (1990).

[38] F-M Dittes, Phys. Rep. 339, 215 (2001).

[39] T. Gorin, F.-M. Dittes, M. Müller, I. Rotter, and T. H. Seligman, Phys. Rev. E 56, 2481 (1997).

[40] S. Albeverio, F. Haake, P. Kurasov, M. Kuś, and P. Šeba, J. Math. Phys. 37, 4888 (1997).

[41] Gnuplot, Linux version 3.7 patchlevel 1 (1999).

[42] M. Lombardi (private communication).

[43] T. Gorin (unpublished). 